\title{
Image Processing and Feature Extraction from a Perspective of Computer Vision and Physical Cosmology
}

Holger Stefan Janzer ${ }^{1}$, Florian Raudies ${ }^{1}$, Heiko Neumann, Frank Steiner

\section{1}

\section{Introduction}

The main conjunction between Physical Cosmology and Computer Vision are images. Commonly structures and objects in those images should be detected and recognized. In this contribution we give a short survey of methods and assumptions used in both disciplines. Applications and illustrative examples of those methods are presented for the fields of Physical Cosmology and Medical Science.

In numerous scientific disciplines and applications areas high-dimensional sensory data needs to be analyzed for the detection of complex structures or for triggering special events. From the beginning the acquisition and analysis of image data formed the subject of image analysis. Nowadays many research disciplines work on the analysis of multi-dimensional images, namely engineering and computer science, physics, mathematics and even psychology. Together they formed the research discipline of Computer Vision (or Computational Vision) which accounts for the interpretation of images and image sequences rather than merely the raw processing of images [1,2]. Computer Vision aims to be an umbrella for tasks that could be classified into:

(a) low-level vision, for example, image enhancement and filtering techniques for image processing;

(b) mid-level vision, for example, segmentation, feature extraction, and the detection of so-called intrinsic scene characteristics, in particular, the relative surface orientation or depth discontinuities with respect to the viewer direction; and

(c) high-level vision to generate and obtain, for example, descriptions of threedimensional surfaces and volumes or the linking to steer a robot through complex terrain $[3,4]$.

The observation of similar approaches and computational methods that have been developed in different disciplines, namely in Computer Vision and Physical Cosmology, have motivated the writing of this contribution. This article high-

1) Corresponding authors. 
lights common assumptions and methods which are used in both the fields of Physical Cosmology and Image Processing/Computer Vision, but which are often not well known in other research communities. At various stages we give insights into current research, beyond the scope of the good, and usual, textbooks as image processing or Physical Cosmology. Here we give a short survey of methods and assumptions utilized for stages of basic image processing and the extraction of meaningful features. Applications and illustrative examples of those methods are presented as images from Physical Cosmology and medical imaging to highlight the broad scope of applicability. The focus of this overview is restricted to the analysis of single images. It would be beyond the scope to discuss approaches of multi-image processing such as in stereo vision or motion analysis. For the interested reader, we refer to standard text books such as, for example, [5].

\subsection{1}

\section{Overall View}

The chapter is organized as follows. We start in Section 10.2 with a brief outline of some definitions and architectural issues in image processing and (low-level) Computer Vision. In addition, a short summary of the background of Physical Cosmology and its relation to image processing is presented in Section 10.3. In Section 10.4 properties of images are discussed, beginning with a sketch of the generation and representation of two-dimensional (2D) images. Image representations are often generated by a projective image acquisition process and involve a proper mapping of the scenic image onto an arbitrarily shaped surface. We briefly sketch some useful transformations often used in applications. Next, several main image properties are introduced. Then a brief overview of basic image characteristics is presented, including basic quantities such as, for example, distribution and correlation measures of image intensities as well as useful spectral measures such as the angular power spectrum and the two-point correlation function. Finally, a generalized framework for image registration is presented. Section 10.5 gives first an overview of the filtering process from systems theory, including a study of filters in Fourier space. Second, some simple methods for the analysis of structures inherent in images are discussed. In Section 10.6 invariance properties are introduced and representations accomplishing these properties are defined. From the perspective of image processing these are statistical moments dealing with continuously valued images. From the perspective of Physical Cosmology we present methods from stochastic geometry dealing with binary structures. We show feature extraction by means of Minkowski Functionals, their generalization as Minkowski Valuations and we present several applications. In Section 10.7 some concluding remarks are given. 
10.2

\section{Background from Computer Vision}

The goal of image analysis is the construction of meaningful descriptions of scenes (with their physical objects) from images and the subsequent interpretation of this description. The result aims at serving functional and behavioral system performances such as, for example, the navigation and collision avoidance of a mobile robot, the sensory-motor control in steering a gripper for object manipulation, or the generation of a scene description in natural language output. For intrinsically 2D scenes, that is scenes with negligible 3D layout, the processing could be depicted in terms of a cascade of sequential processing steps. One operational goal is motivated by the processing and feature extraction for pattern recognition. In a nutshell, such a processing sequence can be summarized by:

- image acquisition, projective mappings, and image enhancement;

- image pre-processing by linear or nonlinear filtering and signal restoration;

- image segmentation and grouping for item aggregation;

- feature extraction or generation of structural image descriptions; and finally

- the classification of shapes and objects.

We will present various examples which highlight the properties and characteristics of images. Our focus is on the primary steps of pre-processing to feature extraction. For example, we start with a display of simple properties based on the statistics of the intensity distribution as well as the joint distribution of intensities in multi-image representations and pairs of image locations and their values. Spectral properties of images are derived using basic integral transforms of image signals, such as the Fourier transform and variants of it. Issues of discrete representations and mappings for projection of planar images onto curved surfaces will be introduced. Basic methods of image processing will be discussed, such as linear and space-invariant filters, which are precursory to the extraction of features from images.

\section{3}

\section{Background from Physical Cosmology}

Cosmology is the scientific study of the large-scale properties of the Universe as a whole. Physical methods are used to understand the origin, evolution and ultimate fate of the entire Universe. The Universe is the entire space-time continuum in which we live, together with all the constituent parts.

Modern cosmology began with Einstein's seminal paper from 1917 [6] in which he applied his general theory of relativity, published only two years earlier, to construct for the first time a relativistic model for the Universe. The Einstein universe is a static one and, furthermore, at the time was consistent with all available astronomical data. Thus it was a great surprise when in 1929 Edwin Hubble observed that distant galaxies fade away, which indicates an expanding Universe. Observa- 
tions show a hierarchy of structures. There are galaxies similar to our Milky Way composed of billions of stars similar to our Sun. Several galaxies form galaxy clusters where gravitational attraction is still dominant over expansion. Further galaxy clusters form galaxy superclusters which form, via filaments, a net-like structure that has large cavities called voids. This structure is called the large-scale structure (LSS) of the Universe. On even bigger scales the Universe is, on average, homogeneous and isotropic. Thereby one can define a mean mass density $\bar{\varrho}(t)$.

On large scales only gravitational interaction is relevant. Thus Einstein's general relativity provides the appropriate theory to describe the Universe as a whole. Homogeneity and isotropy lead to solutions of the Einstein field equations corresponding to a class of universes called Friedmann-Lemaitre universes. These solutions describe the evolution of the local space-time metric and depend on several cosmological parameters, in particular on the energy content of the Universe. Observations suggest, and theory states, that the Universe monotonously expanded since it was generated by the Big Bang $13.7 \times 10^{9}$ years ago.

Had the Universe once been perfectly homogeneous, there would be no structures today. However, the LSS of galaxies, galaxy clusters and galaxy superclusters shows fluctuations of the mass density $\varrho(t, x)$ about the mean $\bar{\varrho}(t)$ measured by the density contrast $\Delta \varrho(t, x)=(\varrho(t, x)-\bar{\varrho}(t)) / \bar{\varrho}(t)$. These fluctuations are caused by primordial density fluctuations derived from the initial conditions at the Big Bang. After the Big Bang the ingredients of the Universe have undergone several phase transitions. 380000 years after the Big Bang, called the decoupling age $t_{\text {rec }}$, matter and radiation decoupled. Thereby the detectable radiation background called the cosmic microwave background (CMB) resulted from free streaming photons. Note that the CMB is an almost perfect isotropic radiation on the celestial sphere which satisfies the quantum mechanical law of temperature radiation, that is Planck's law, with a mean temperature of $\bar{T}=2.725 \mathrm{~K}$ with an extraordinary precision and possessing tiny temperature deviations from isotropy of relative order of $\Delta T(\theta, \phi)=(T(\theta, \phi)-\bar{T}) / \bar{T} \propto 10^{-5}$ only. These fluctuations are highly correlated ${ }^{2)}$ with the mass density contrast $\Delta \varrho\left(t_{\mathrm{rec}}, x\right)$ of the entire early universe at the decoupling age $t_{\text {rec }}$. They are interpreted as the seed of todays observed structures and can be understood as a kind of projected snapshot from the entire early universe at decoupling age. Thereby the CMB represents one of the most powerful tools in cosmology and is the oldest accessible information with possibilities today. The fluctuations of the CMB are shown in Figure 10.1.

Due to relativistic effects, that is, the finite speed of light, information of distant objects can only be received from past events. Furthermore, the expansion of the Universe prohibits the access to the entire Universe. That part which is accessible to observations is called the observable Universe. Since we cannot perform experiments with many universes, for example, we cannot repeat the Big Bang, cos-

2) In the full relativistic description there is a dependence on the total energy content. In the standard model there is radiation, baryonic matter, dark matter and dark energy. One distinguishes between primary effects, that is, effects at decoupling age, and secondary effects, that are effects during propagation from decoupling age until the observation time. 


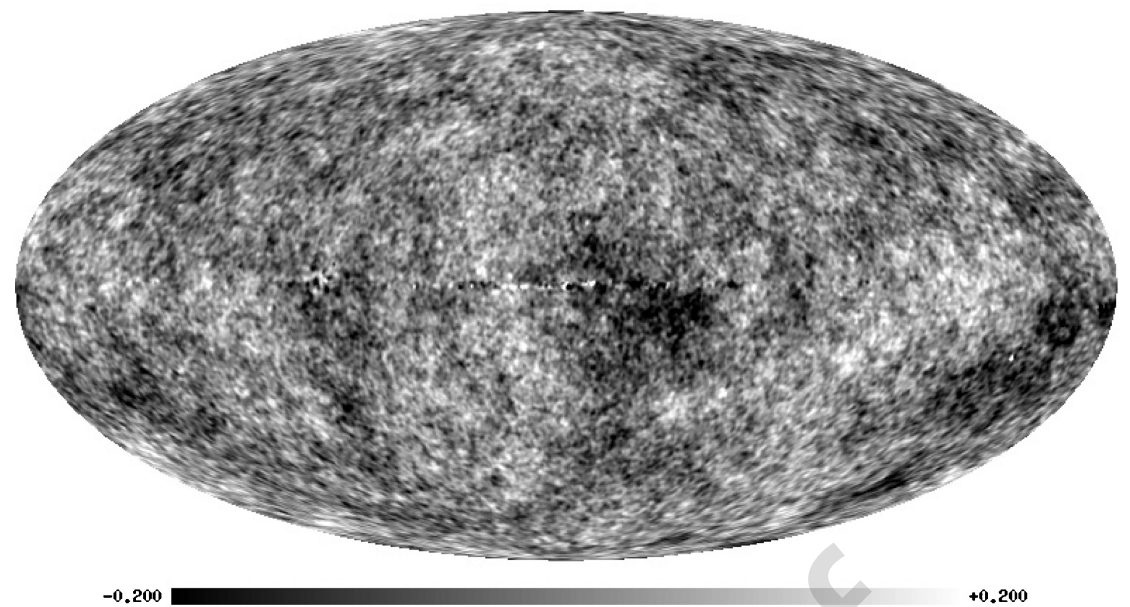

Fig. 10.1 Cosmic microwave background $(\mathrm{CMB})-$ Fluctuations $(T(\theta, \phi)-\bar{T}) \mathrm{mK}$ of the CMB measured by the NASA satellite Wilkinson Microwave Anisotropy Probe (WMAP) [7]. Dark indicates colder regions and bright hotter regions than the mean temperature of $\bar{T}=2.725 \mathrm{~K}$. These structures detected on the celestial sphere are interpreted as the seeds of today's observed structures and can be understood as a kind of snapshot of the entire early Universe at the decoupling age. The image is displayed in Mollweide projection (see Section 10.4.1) and has a resolution of $12 \times 512^{2}$ pixels in HEALPix pixelization [8].

mology is based on observations concerning just one universe, that is the one in which we happen to live. For that reason large-scale computations are performed simulating a large ensemble of universes. We are forced to extrapolate the laws of physics to extreme conditions where, in principle, they may no longer apply. For comparison and distinction of complex outputs, methods of image processing and feature extraction are of major interest in cosmology as will be illustrated below.

\section{4}

\section{Image Formation and Characterization}

Images are acquired in different applications for further analysis of their structural content for evaluation or steering and control purposes. At the sensory level of (discrete) signal generation an image can be described as a structural distribution of intensity or grey values. From a different point of view, namely statistical physics, the image values can be considered as an observations of stochastic variables. Both perspectives lead to essentially the same definitions of such basic image properties. Here, we will first present some basic formal methods to describe the generation and representation format for 2D images. Next, we sketch some basic image properties, namely their representation, the characteristics, and the registration of images. To study the characteristics, we model an image as an observation of a stochastic process (as sketched above), which is also the basic model in Phys- 
ical Cosmology where it is assumed that the initial conditions of the Universe are described by a homogeneous and isotropic Gaussian random field. Throughout the article $u(x)$ denotes a field of scalar values which can be interpreted in two ways, namely, as intensity or probability distributions. Such scalar values in a field are addressed for the spatial position of a vector in an arbitrary space. Multiple intensity values, which are measured by different sensory channels or registration methods, but in a spatially registered way, can be represented as a vector field $\boldsymbol{u}(x)$. In other words, such an image, at a spatial position $u(x)$, contains a vector of dimension 2 , 3 , or $n$, depending on the number of registered sensory channels. Examples are images from magnetic resonance imaging (MRI) combining spin relaxation, T1 and T2, images taken by a color camera measuring in different bands of wavelength sensitivity as red, green, blue (RGB), or a scalar image field of intensity values combined with another field of derived features, such as local variances measured in a local image neighborhood.

\subsection{1}

\section{Generation and Representation of 2D Images}

Our focus in this article will be on two-dimensional (2D) images which are acquired from some environmental measurement space of arbitrary dimension. In the case of the three-dimensional (3D) space of an outdoor scene the image acquisition process can be formally described by a projective transform. Most often the projection results in a 2D Cartesian coordinate system commonly named image plane. In Physical Cosmology, instead, an image can be defined on a unit sphere. In such cases the mapping of the (image) plane onto the curved surface can be described by a geometric transform. If the topology of the two such surfaces is identical then the mapping can be inverted. In the case when the image acquisition is distorted, for example due to some geometric lens distortion, the proper registration is also described by a proper warping transform. Here, we briefly sketch some projective as well as mapping transformations. A more complete overview is given in [9].

\subsubsection{1}

\section{Perspective Projection}

For the perspective projection, a point in $3 \mathrm{D}$ space $x \in \mathbb{R}^{3}$ is projected in $2 \mathrm{D}$ space $y \in \mathbb{R}^{2}$, for example, representing an image plane, by

$$
\left(y_{1}, y_{2}\right)=\left(x_{1}, x_{2}\right) \cdot f / x_{3},
$$

where the third component is omitted because it equals the constant $f$. The geometric interpretation is that arbitrary points (normally $x_{3}>f$ ) are projected onto an image plane positioned at a positive distance $x_{3}=f$ from the coordinate center $(0,0,0)$. If, instead, the distance from the projection center is taken to be negative, that is $-f$, the projection resembles a pinhole projection. A key characteristic is that the resulting image is upside down (negative image) while the former case yields a positive image. It should be noted that, in the extreme case of very distant scene 
points with $x_{3} \gg f$, for small objects relative to the field of view, and with only minor relative depth variations, the perspective projection can be approximated by an orthographic projection, namely $\left(\gamma_{1}, \gamma_{2}\right)=\left(x_{1}, x_{2}\right)$.

\subsubsection{2}

\section{Stereographic Projection}

In various applications, specific surface properties, such as their 3D orientation, need to be represented in a flat space in order to build proper data structures. A useful approach is to map the spherical hemisphere of surface normals visible from the observer's viewpoint onto a tangential plane positioned in the sphere's north pole. If the center of projection is shifted into the south pole, then the upper hemisphere of the unit sphere is mapped into a circle of radius two (stereographic projection; [10]). Though this mapping is complete it also has some shortcomings, namely that only one-half of the sphere is projected and that the projection is not area-preserving.

\subsubsection{3}

\section{Mollweide Projection}

Generally, images can be defined on arbitrary surfaces (see also Section 10.4.4). Examples are the surface of the earth, which is ideally defined on a unit sphere, or satellites which measure over the celestial sphere. Therefore, images defined on the sphere play a special role. An approach that overcomes the limitations of the stereographic projection is the Mollweide projection,

$$
\begin{aligned}
& y_{1}=(2 \sqrt{2} / \pi) \Phi \cos (\Psi / 2) \\
& y_{2}=\sqrt{2} \sin (\Psi / 2) \quad \text { with } \quad \Psi+\sin \Psi=\pi \sin \Theta \text { and } \\
& \Phi=\arg \left(x_{1}+i x_{2}\right), \quad \Theta=\arctan \left(x_{3} / \sqrt{x_{1}^{2}+x_{2}^{2}}\right)
\end{aligned}
$$

where $\Phi \in(-\pi,+\pi]$ denotes the longitude from the central meridian, $\Theta \in(-\pi / 2$, $+\pi / 2$ ) denotes the latitude, and $\Psi$ denotes only an auxiliary angle. This projects the surface of the total unit sphere onto a plane forming an ellipse, where the major axis is twice as long as the minor axis. Additionally, this projection is area preserving. This property can be easily checked if one integrates over an arbitrary

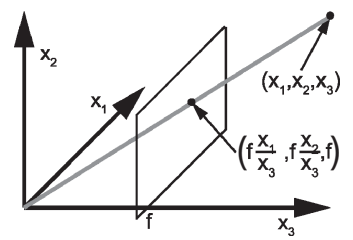

(a)

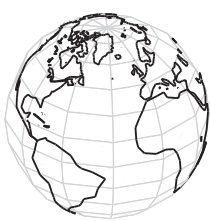

(b)

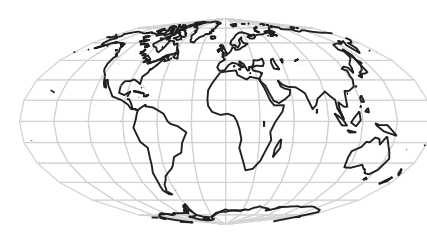

(c)

Fig. 10.2 Projections: (a) Perspective projection of the point $\left(x_{1}, x_{2}, x_{3}\right)$ onto the image plane positioned at $f$. (b) Continental contour lines of the earth. (c) Area-preserving Mollweide projection for the continental contour lines. 
surface patch on the sphere and on the plane, and shows that the calculated areas are equal in size. Figure 10.2c shows the projection for the continental contours of the earth.

10.4.2

\section{Image Properties}

In each image representation the following key properties can be identified: (i) The space or surface where the image is defined (see methods for projections in Section 10.4.1); (ii) the number of quantization levels; (iii) the resolution; and (iv) the scale. Although, the scale of an image is intertwined with the resolution, we discuss these two properties separately. Figure 10.3 depicts all these properties.

\subsubsection{1}

\section{Quantization Property}

The quantization levels are defined by the range of the data values. This property is determined by two criteria. First, the range of the acquisition sensor, and

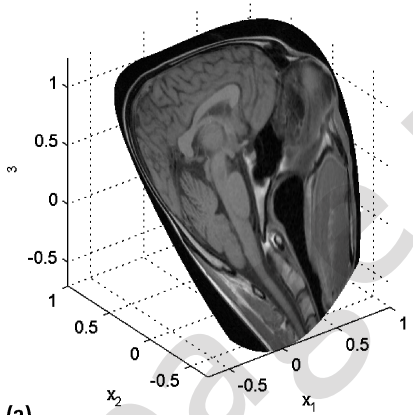

(a)

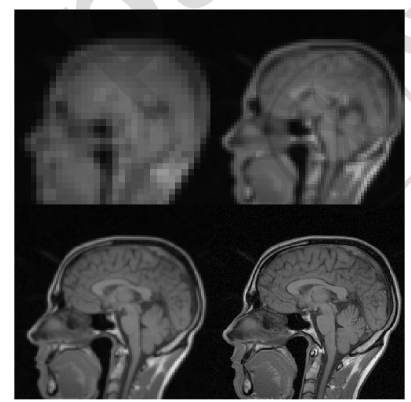

(c)

Fig. 10.3 Image properties: (a) 2D magnetic resonance image mapped onto an arbitrary surface, thus the image coordinates depend on the surface geometry. (b) Same image visualized with 4, 8, 16 and 32 quantization levels. (c) Image with a resolution of $32 \times 32 \mathrm{px}$,

(b)
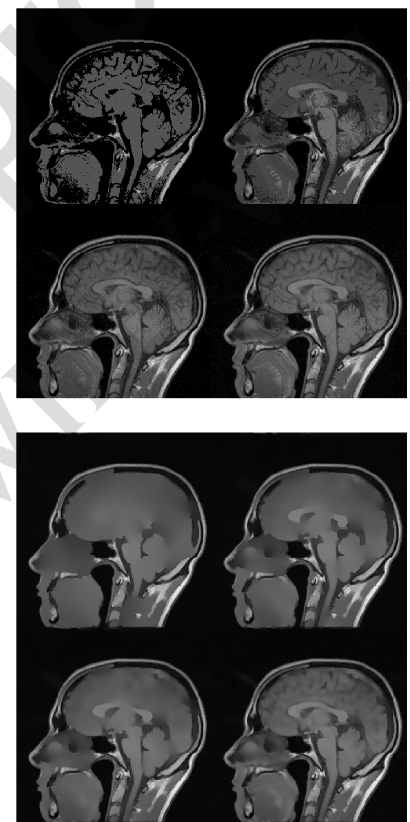

$64 \times 64 \mathrm{px}, 128 \times 128 \mathrm{px}$, and $256 \times 256 \mathrm{px}$ (original). (d) Scales of nonlinear isotropic diffusion of the image for 1000, 100, 50, and 10 iterations $(\lambda=0.02, \sigma=1.5$, parameters referring to [11]). 
second the storage format. Ordinary formats have 8 Bits, and thus 256 quantization levels. For color images each channel of the three channels in an RGB model could be quantized into 8 Bits, consequently having 24 Bits. Note, that the quantization always produces an error of discretization, which depends on the number of quantization levels and the sampling of the quantization interval.

\subsubsection{2}

\section{Spatial Resolution Property}

The upper spatial resolution is limited by the quality of the sensor, and the lower resolution is determined by the smallest object in the image which should be properly represented. Generally this depends on further processing tasks, for example, the successful recognition of a small object. Additionally the arrangement of pixels can be done in different ways for an image which is defined on a sphere the choice is not particularly obvious. Therefore, we refer to the HEALPix [8] pixelization technique on a sphere which possesses many features of a cartesian pixelization on a plane.

\subsubsection{3}

\section{Scale Space Property}

Scale spaces are used to represent the inner structure of an image with different levels of detail. On the finest level, all objects and structures are visible. In contrast, larger scales combine fine neighboring structures and subsume them to single objects. A general technique for the construction of scale spaces is the diffusion process [11],

$$
\frac{\partial}{\partial t} u(x, t)=\Delta[\varrho(u ; x, t) \nabla u(x, t)]
$$

with $u$ denoting the current diffused image at each location $x$ for an internal time $t$, or scale. $\varrho$ denotes the diffusion parameter, which determines the local rate of diffusion. In the simplest case, called homogenous diffusion, $\varrho$ is a constant and does, not depend either on space or time, or on the actual solution $u(x, t)$. For this type of diffusion an analytical solution can be derived based on the Green's function approach which leads to a Gaussian kernel with $\sigma=\sqrt{2 t}$. An image constructed with this type of diffusion could also be defined by a corresponding resolution, which means smoothing the image with the same Gaussian kernel as for the diffusion process. Other types of diffusion for the construction of different scales, have results which cannot be derived by Gaussian smoothing. In particular, the results in Figure 10.3 (c) and (d) for resolution and scale are different. Here, a nonlinear isotropic diffusion is used for the construction of different scales, where $\varrho$ depends on the actual solution $u(x, t)$ and is a scalar. In image processing $\varrho$ is regulated by the structure of the actual solution $u(x, t)$, for example, the image gradient. 
10.4.3

\section{Basic Image Characteristics}

For the analysis of image characteristics an image can be defined as the observation of a stochastic process in three ways. First, the image is modeled as the outcome of one random variable (RV); second, as one observation of a random field (RF), and third as a series of RVs. Each distinct modeling allows the study of distinct image characteristics which contain information of the structure and distribution of image intensities. An overview of characteristics and modeling is given in Table 10.1.

\subsubsection{1}

\section{Histogram}

Assume that all intensities contained in an image are continuous and the outcome of a single RV $\boldsymbol{X}$. Additionally, the probability distribution function of $\boldsymbol{X}$ is $u(x)$ for all $x \in \mathbb{R}$ of the random space. With this formalism the distribution of intensities in an image can be expressed by the normalized histogram

$$
H_{\mathrm{N}}\left(B_{\varepsilon}\right):=P\left(X \in B_{\varepsilon}\right)=\int_{x \in B_{\varepsilon}} u(x) \mathrm{d} x
$$

with $B_{\varepsilon}:=\{x \mid b-\varepsilon / 2 \leq x<b+\varepsilon / 2\}$, which equals the probability distribution function for $\varepsilon \rightarrow 0$. For images with continuous intensities the histogram has bins counting an interval of intensities which have only a finite number of levels. In this case $\varepsilon$ defines the width of these bins. Figure 10.4a shows histograms of three image features. For the cumulative normalized histogram

$$
H_{\mathrm{N}, \mathrm{C}}(b):=F_{u}(b)=\int_{-\infty}^{b} u(x) \mathrm{d} x
$$

these bins are not necessary and one integrates from the lowest possible intensity $-\infty$ to the intensity level $b$. This histogram equals the probability mass function $F_{u}$ of the RV $\boldsymbol{X}$.

\subsubsection{2}

\section{Covariance and Correlation}

For two images modeled by RVs $X$ and $Y$ a comparison on the basis of their statistical behavior can be achieved by the covariance

$$
\operatorname{Cov}_{X, Y}=\langle(X-\langle X\rangle)(Y-\langle Y\rangle)\rangle=\langle X Y\rangle-\langle X\rangle\langle Y\rangle
$$

Tab. 10.1 Formalism for the modeling of an image and the achieved image characteristics.

\begin{tabular}{llll}
\hline Model & Single RV & RF & Series of RVs \\
\hline One input & histogram & $\begin{array}{l}\text { co-occurrence, Fourier transformation, } \\
\text { two-point correlation } \\
\text { joint histogram, correlation }\end{array}$ & $\begin{array}{l}\text { joint distribution, } \\
\text { Fourier transformation } \\
-\end{array}$
\end{tabular}




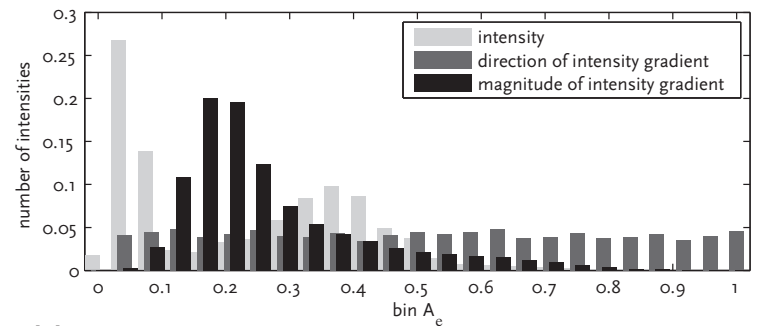

(a)

Fig. 10.4 Image characteristics: (a) Histogram for image features, the intensity, direction, and magnitude of intensity gradient are from Figure $10.3 \mathrm{c}$ original (25 bins). Therefore the direction and magnitude are normalized to the unit interval. The magnitude has a unimodal histogram (one peak), the intensity a bimodal histogram (two peaks), and the direction is

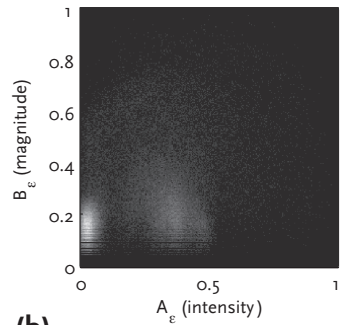

(b)

almost equaly distributed. (b) Joint histogram between intensity and gradient magnitude (256 bins). The two peaks in the histogram are the overlay between the unimodal and multimodal histograms, where the peaks are located at the same bin numbers as in the histograms. For visualization the square root of the joint histogram is shown.

where $\langle\cdot\rangle$ denotes the average over all observations of the RV. This definition is lacking for the dependence of the interval of Cov on the interval of outcomes from $X$ and $Y$. A normalized variant is the correlation

$$
\operatorname{Cor}_{X, Y}=\operatorname{Cov}_{X, Y} / \sqrt{\operatorname{Var}(X) \operatorname{Var}(\mathbf{Y})} \in[-1,+1] \text {, }
$$

where Var denotes the variance of the individual random variables. The division by the variances of $\boldsymbol{X}$ and $Y$ normalizes the interval to $[-1,+1]$ independently of the input intervals. Correlation-based techniques are used in many fields of image processing; for example, for template matching, flow/stereo estimation and image registration $[12,13]$. Generally, the goal is the determination of correspondences between the same sub-image in two images with temporal or spatial coherence.

\subsubsection{3}

\section{Joint Histogram}

Both characteristics (the normalized histogram and the correlation) do not include any information about the spatial distribution of intensities. All intensities are outcomes of the same single RV not bound on any spatial position in the image. On the contrary a dependence can be modeled by RFs $X\left(x_{1}, x_{2}\right)$, containing one RV for each spatial position $\left(x_{1}, x_{2}\right)$ in the image (here 2D). For two RFs $X\left(x_{1}, x_{2}\right)$ and $Y\left(x_{1}, x_{2}\right)$ with their corresponding probability distribution functions $u$ and $v$, the joint normalized histogram is

$$
\begin{aligned}
H_{\mathrm{J}}\left(A_{\varepsilon}, B_{\varepsilon}\right) & =\iint P\left(X \in A_{\varepsilon} \wedge Y \in B_{\varepsilon}\right) \mathrm{d} x_{1} \mathrm{~d} x_{2} \\
& =\iiint_{x \in A_{\varepsilon}} \int_{Y \in B_{\varepsilon}} u\left(x_{1}, x_{2} ; x\right) v\left(x_{1}, x_{2} ; \gamma\right) \mathrm{d} x \mathrm{~d} \gamma \mathrm{d} x_{1} \mathrm{~d} x_{2},
\end{aligned}
$$

where the outer two integrals operate over the total image domain, and the inner two integrals over the bin intervals $A_{\varepsilon}$ and $B_{\varepsilon}$. Note, that a separate probability 
distribution function $u$ exists for each position $\left(x_{1}, x_{2}\right)$ of the RF, and $x$ denotes the argument of the function $u$, as in the 1D case $u(x)$. In the joint histogram, paired intensities according to the bins $A_{\varepsilon}$ and $B_{\varepsilon}$ are voted, where the intensity pair is located at arbitrary positions $\left(x_{1}, x_{2}\right)$ in the two RFs $X$ and $Y$. In Figure 10.4 (b) a joint histogram between the feature channels intensity and magnitude of the intensity gradient is shown.

\subsubsection{4}

\section{Co-occurrence}

For images with multiple channels, as for example images acquired by multispectral sensors or color images, a joint histogram describes the correlation between the intensity distributions of different channels. The joint occurrence of values within one image or channel can be quantified by the co-occurrence matrix

$$
\operatorname{Co}\left(A_{\varepsilon}, B_{\varepsilon}\right)=\iiint_{x \in A_{\varepsilon}} \int_{\gamma \in B_{\varepsilon}} u\left(x_{1}, x_{2} ; x\right) u\left(T\left(x_{1}, x_{2}\right) ; \gamma\right) \mathrm{d} x \mathrm{~d} \gamma \mathrm{d} x_{1} \mathrm{~d} x_{2} .
$$

Here, the first RF $X\left(x_{1}, x_{2}\right)$ is defined by the image and the second RF $X_{T}\left(x_{1}, x_{2}\right)$ by a spatial transformation $T\left(x_{1}, x_{2}\right)$ of the first RF. This characteristic highlights periodic structures and shifts of regions within one image.

\subsubsection{5}

\section{Fourier Transformation of One Observation of an RF}

Many applications in image processing profit from the analysis of images in the Fourier space, especially the study of the effectiveness of filters (see Section 10.5). The Fourier transformation

$$
\begin{aligned}
& \hat{u}\left(k_{1}, \ldots, k_{d} ; x\right)=\int_{\mathbb{C}} \ldots \int_{\mathbb{C}} u\left(x_{1}, \ldots, x_{d} ; x\right) \exp \left(-i \sum_{l=1}^{d} k_{l} x_{l}\right) \mathrm{d} x_{1} \ldots \mathrm{d} x_{d}, \\
& u\left(x_{1}, \ldots, x_{d} ; x\right)=\frac{1}{(2 \pi)^{d}} \int_{\mathbb{C}} \ldots \int_{\mathbb{C}} \hat{u}\left(k_{1}, \ldots, k_{d} ; x\right) \exp \left(i \sum_{l=1}^{d} k_{l} x_{l}\right) \mathrm{d} k_{1} \ldots . \mathrm{d} k_{d},
\end{aligned}
$$

from the spatial domain $u$ into the temporal domain $\hat{u}$, is again based on the formalism of the RF, where one concrete observation of the RF is transformed. After the transformation into the Fourier space, $\hat{u}$ is a complex number. Therefore, $\hat{u}$ can be analyzed in phase $\Phi$ and amplitude $A$

$$
\Phi=\arg (\hat{u}), \quad \text { and } \quad A=|\hat{u}| .
$$

In Figure 10.5b the amplitude of the transformed input image a is shown. The corresponding inverse transformation of a filtered version is depicted in Figure 10.5d. For images the information about the spatial locality of structure is stored in the phase, and the information about the general periodicity is represented within the amplitude. To highlight this property consider a shift of the image in the spatial domain, therefore only the phase is influenced, not the amplitude. Thus, no information of the locality is included in the amplitude. 


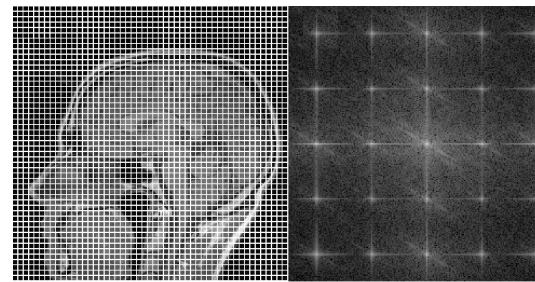

(a)

(b)

Fig. 10.5 Fourier and inverse Fourier transformation: (a) Input image again from Figure 10.3b (original) with superimposed regular grid. (b) Fourier spectra (amplitude) of the image with characteristic peaks representing the grid and their multiples. (c)

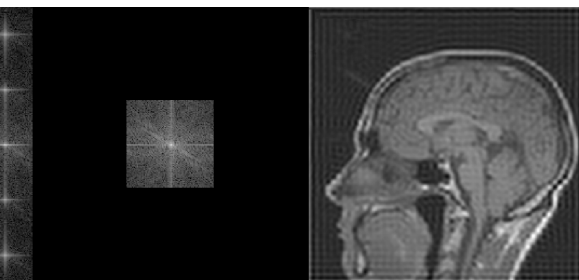

(c)

(d)

Boxed version of the fourier spectra. This has a box filter, where frequencies representing the first multitude of the grid are cut off. For visualization the square-root of the spectra is plotted. (d) Inverse Fourier transformation of boxed spectra multiplied by the box filter.

\subsubsection{6}

\section{Fourier Transformation of a Common Distribution Function}

In some cases the stochastic process is defined by a series of $\operatorname{RVs}\left(X_{1}, \ldots, X_{d}\right)$, and this models the dimensionality of the image. For example, an image is defined as the outcome of a $d \mathrm{D}$ normally distributed RV. Analogous to the Fourier transformation of RF, here the transformation is defined on the basis of the common density distribution function $u\left(x_{1}, \ldots, x_{d}\right)$ which equals the product of the single distribution function $u\left(x_{i}\right)$ if the RVs are independent. The Fourier transformation

$$
\hat{u}(k)=\int_{\mathbb{C}^{d}} u(x) \exp (-i k x) \mathrm{d} x, \quad u(x)=\frac{1}{(2 \pi)^{d}} \int_{\mathbb{C}^{d}} \hat{u}(k) \exp (i k x) \mathrm{d} k
$$

where $x, k \in \mathbb{R}^{d}$ is the joint characteristics function of the RVs $X_{1}, \ldots, X_{d}$. In other terms this is the expected value $\hat{u}=\left\langle\exp \left(-i \sum_{l=1}^{d} k_{l} X_{l}\right)\right\rangle$ of the series of RVs in respect to the Fourier base. $\hat{u}$ can be interpreted as the probability distribution function of new RVs $\hat{X}_{1}, \ldots, \hat{X}_{d}$, which are also independent, in fact, of the orthogonality of the Fourier base.

\subsubsection{7}

\section{Two-Point Correlation Function and Power Spectrum}

In cosmology, images of homogeneous and isotropic RFs are often studied. Here, characteristics of length scale or separation distance are of special interest. The power spectrum is given by an average over the Fourier modes $\hat{u}(k)$ with a wave number $k=|k|$ of the field ${ }^{3)} u(x)$ with $x \in \mathbb{R}^{3}$. In configuration space, a field can be quantified by the two-point correlation function

$$
\xi(r):=\langle u(x) u(x+r)\rangle
$$

3) Note that we omitted the $x$ for one realization of the RF $u(x, x)$ at the position $x$, because cosmological observations always display one realization. 
where the average $\langle\cdot\rangle$ is taken over all positions $x$ and all orientations of the separation vector $\boldsymbol{r}$, assuming homogeneity and isotropy. Hence there is only a dependence on $r=|r|$. The two-point correlation function of a field on a sphere is

$$
C(\vartheta):=\left\langle u(\theta, \phi) u\left(\theta^{\prime}, \phi^{\prime}\right)\right\rangle,
$$

where now the average $\langle\cdot\rangle$ is taken over all pairs of $(\theta, \phi)$ and $\left(\theta^{\prime}, \phi^{\prime}\right)$ which are separated by the angle $\vartheta$. Again a power spectrum

$$
C_{l}:=\left\langle\left|a_{l m}\right|^{2}\right\rangle=\frac{1}{2 l+1} \sum_{m=-l}^{l}\left|a_{l m}\right|^{2}
$$

can be defined, where $a_{l m}$ are the complex coefficients obtained from an expansion into spherical harmonics $Y_{l m}(\theta, \phi)$ due to $u(\theta, \phi)=\sum_{l=0}^{\infty} \sum_{m=-l}^{l} a_{l m} Y_{l m}$. Here $l$ parameterizes the separation scale and $m$ the direction. In cosmological applications the so-called angular power spectrum

$$
\delta T_{l}^{2}:=\frac{l(l+1)}{2 \pi} C_{l}
$$

is used. Note that, in the case of statistical homogeneity and isotropy, a two-point correlation function can be obtained by a transformation from its corresponding

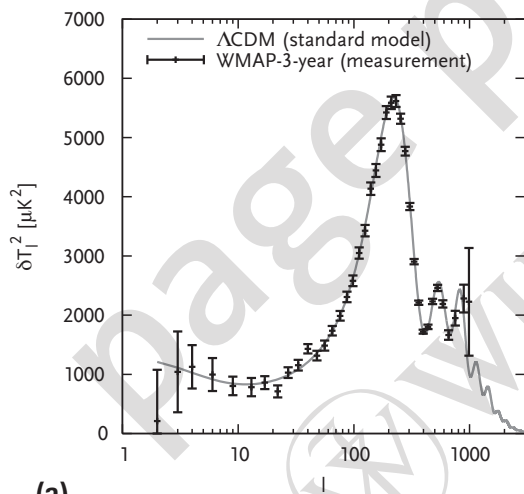

(a)

Fig. 10.6 Statistical quantities of the CMB: (a) Angular power spectrum $\delta T_{l}^{2}$ of the observed fluctuations in the cosmic microwave background (CMB) measured by the Wilkinson Microwave Anisotropy Probe (WMAP) [7]. The error bars include measurement errors and the statistical variance. These characteristics are very sensitive, i.e. the peak positions and peak heights, to cosmological parameters. Independent cosmological determinations of the cosmological parameters are in excellent agreement with the best-fit standard model ACDM (gray line). The systematic deviations on largest scales (small $l$ ) cannot be explained

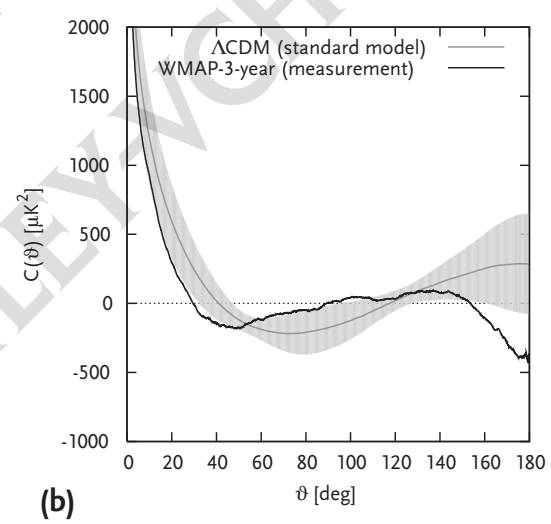

by the standard model and are possible indications of a finite Universe [14]. (b) Twopoint correlation function $C(\vartheta)$ of the best-fit standard model $\Lambda$ CDM (dark gray line and the statistical variance as light gray area) and measurements of WMAP (black line). These characteristics highlight the largest scales $\left(\vartheta \approx 180^{\circ} / l\right)$ where the explanation by the standard model is limited. Going beyond the standard model, recent work shows [14], that beside the suppression of $\delta T_{l}^{2}$ on small $l$ the shape of this characteristic can be reproduced with high confidence, by studying universes with a finite spatial extension. 
power spectrum and vice versa. These characteristics carry the same information, but highlight different separation scales and thus different cosmological features. In Figure 10.6 the angular power spectrum and the two-point correlation function of the measured cosmological microwave background (CMB) is shown where now $u(\theta, \phi)=T(\theta, \phi)-\bar{T}$ (see Section 10.3). In addition to omitting the constant term (monopole) with $l=0$, which is equivalent to $\bar{T}$, the dipole with $l=1$ is, also omitted due to a superimposed dipole generated by the relative motion of the observer to the CMB.

\subsection{4}

\section{Image Registration}

The problem in image registration is to find a mapping between a reference image $u^{\text {ref }}$ and a template image $u^{\text {tem }}$. Formally, the problem is to determine a transformation $\phi$ applied to the template minimizing the difference to the reference image. This is a minimization problem which could include additional constraints, for example, the exact mapping of specific parts within the template and corresponding parts in the reference image. Here, we describe a generalized framework formerly introduced in [15] which is based on variational techniques. Let us define the optimization problem

$$
E(\phi):=D\left(u^{\mathrm{ref}}, u^{\mathrm{tem}} ; \phi\right)+\alpha S(\phi)+\beta C^{\mathrm{soft}}(\phi) \stackrel{\phi}{\rightarrow} \min , \quad C^{\text {hard }}(\phi)=0,
$$

which contains three main terms: (i) a data term $D$; (ii) a smoothness term $S$; and (iii) a (soft) constraint term $C^{\text {soft }}$. Additionally, hard constraints $C^{\text {hard }}$ can be included by side conditions. The parameter $\alpha$ steers the smoothness and $\beta$ controls the influence of additional constraints, respectively. In Figure 10.7 the functionality of the three main terms is depicted. The task is to find a transformation $\hat{\phi}$ such that $E(\hat{\phi})$ is minimal, considering the side conditions. A restricted class of possible transformations are affine transformations, including translation $t$, rotation $r$, scaling $c$, and shear $s$. For these transformations each spatial position $x \in \mathbb{R}^{3}$ is transformed into projective space by $\Theta(x)=\gamma=\left(x_{1}, x_{2}, x_{3}, 1\right)$. Inverse transformation $\Theta^{-1}$ is realized by $x=\left(\gamma_{1}, \gamma_{2}, \gamma_{3}\right) / \gamma_{4}$, if the fourth component of $\gamma$ is not equal

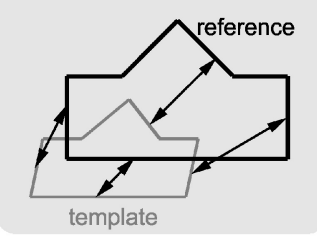

(a)

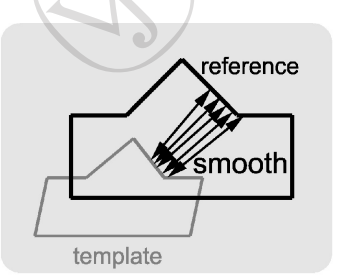

(b)

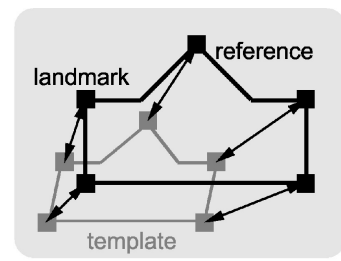

(c)

Fig. 10.7 Image registration: (a) The problem in image registration is to find a mapping between the template and the reference image. (b) Additionally, smoothness for the solving transformation $\phi$ could be required, and (c) landmarks should be matched best for soft constraints and exact for hard constraints. 


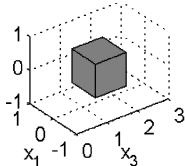

(a)

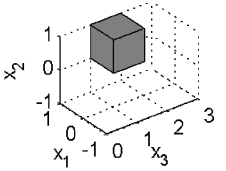

(b)

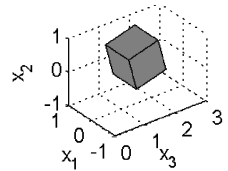

(c)

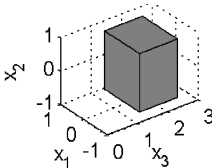

(d)

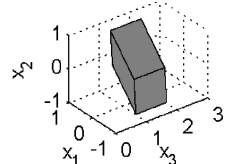

(e)

Fig. 10.8 Affine transformations: (a) Reference for the transformations. (b) Translation for $t=(0.5,0.5,0)$. (c) Rotation for $\boldsymbol{r}=(15,0,0)$ deg. (d) Scaling for $\boldsymbol{c}=(1.5,1.75,1.25)$. (e) Shear for $s=(85,0,0)$ deg.

to zero. Affine transformations in the projective space are realized by a sequential chained multiplication of transformation matrices

$$
\begin{aligned}
\phi(t, r, c, s ; \gamma) & =\left(\begin{array}{cccc}
1 & 0 & 0 & t_{1} \\
0 & 1 & 0 & t_{2} \\
0 & 0 & 1 & t_{3} \\
0 & 0 & 0 & 1
\end{array}\right)\left(\begin{array}{cccc}
1 & 0 & 0 & 0 \\
0 & \cos r_{1} & \sin r_{1} & 0 \\
0 & -\sin r_{1} & \cos r_{1} & 0 \\
0 & 0 & 0 & 1
\end{array}\right) \cdots \\
& \left(\begin{array}{ccccc}
c_{1} & 0 & 0 & 0 \\
0 & c_{2} & 0 & 0 \\
0 & 0 & c_{3} & 0 \\
0 & 0 & 0 & 1
\end{array}\right)\left(\begin{array}{cccc}
1 & \cot s_{1} & 0 & 0 \\
1 & 1 & 0 & 0 \\
0 & 0 & 1 & 0 \\
0 & 0 & 0 & 1
\end{array}\right) \cdots \psi,
\end{aligned}
$$

where we indicated all three rotational matrices by the rotation around the $x_{1}$-axis, and all three shearing matrices by the shear in the $x_{1} x_{2}$-plane. Figure 10.8 depicts examples of those transformations. More advanced transformation techniques can be found in [9].

For global affine transformations the parameters $t, r, c, s \in \mathbb{R}^{3}$ are defined only once for the domain, while local affine transformations exist for each spatial position $t(x), r(x), c(x)$, and $s(x)$. This approach is very extensive introducing twelve unknowns for each spatial position. A simplification assumes constant transformations in small regions within the total spatial image domain.

10.4.4.1

\section{Data Term}

The data term $D$ compares the (gray) values of the reference image $u^{\text {ref }}$ (or some extracted feature measure, such as edges, corners, etc.) with the values of the transformed template image $u^{\text {tem }}$. In this case several distance measures could be applied. An intuitive measure of distances is the sum of squared distances (SSD):

$$
D\left(u^{\mathrm{ref}}, u^{\mathrm{tem}} ; \phi\right)=\int\left[u^{\mathrm{ref}}(x)-u^{\mathrm{tem}}\left(\Theta^{-1}(\phi[t, \boldsymbol{r}, \boldsymbol{c}, \boldsymbol{s} ; \Theta(x)])\right)\right]^{2} \mathrm{~d} \boldsymbol{x} .
$$

This distance assumes that the intensities of corresponding values in $u^{\text {ref }}$ and $u^{\text {tem }}$ are equal. If this assumption is not satisfied, correlation-based methods could be applied, which assume a unimodal distribution of intensity values. For images with a multimodal histogram, mutual information (MI) related measures could be used, 
which are based on the joint intensity histogram. In general, the data term $D$ is also called an external force, because this term is mainly driven by the intensity values of the template and reference.

\subsubsection{2}

\section{Smoothness Term}

In contrast to the data term, the smoothness term $S$ is defined by the mapping $\phi$ which constitutes an internal force by imposing a condition on the set of possible solutions. The key observation is that in this framework any smoother which is Gârteaux-derivable could be applied [15]. Because of its similarity with the diffusion equation in Section 10.4.2 we present as a smoothness condition, the diffusion smoothness

$$
S(\phi)=\int\|\nabla \phi(t, r, c, s ; \Theta(x))\|_{2}^{2} \mathrm{~d} x .
$$

Here the integral, which ranges over the total image domain, induces global smoothness onto the mapping function $\phi$, by squaring and summing up all firstorder partial derivatives of $\phi$ according to the spatial change of the variables $t, r, c$, and $s$. Thus, each strong change in $\phi$ causes a high derivative, which is unwanted and therefore penalized.

\subsubsection{3}

\section{Constraint Term}

Finally, we discuss the inclusion of extra constraints that need to be achieved by the optimized solution. Assume two sets of landmarks, the first set defined in the reference image $\left\{x_{l}^{\mathrm{ref}}\right\}_{l=1 \ldots m}$, and the second set defined in the template image $\left\{x_{l}^{\text {tem }}\right\}_{l=1 \ldots m}$, where correspondence between the landmarks is expressed by the same index. A soft constraint term can be formalized by

$$
C^{\mathrm{soft}}(\phi)=\sum_{l=1}^{m}\left\|\Theta^{-1}\left(\phi\left[t, r, c, s ; \Theta\left(x_{l}^{\mathrm{tem}}\right)\right]\right)-x_{l}^{\mathrm{ref}}\right\|_{2}^{2} .
$$

This constraint enforces the transformed landmarks of the template to be closely matched with the landmarks of the reference $x_{l}^{\text {ref }}$, but deviations are possible. In contrast, for the hard constraint $C^{\text {hard }}$ a match should be exact.

\section{5}

\section{Methods of Image Processing}

In this section we discuss some approaches for pre-processing image signals utilizing a filtering process. Many methods in image processing utilize mechanism that can be described in terms of linear systems theory $[2,9,16]$. Filters can be defined according to their functional purpose, for example, smoothing by elimination of high-frequency content, or discontinuity detection by extracting high-frequency 
content. We briefly summarize the properties of linear systems and display Gaussian smoothing filters and some related derivative operations. So-called Gabor filters define band-pass operations for combined smoothing and discontinuity detection having localized spectral windowing properties. We also show how a bank of filters can be constructed. Finally, we briefly present approaches to nonlinear filtering as well as approaches that help to detect localized key points which obey local 2D image structure properties.

\subsection{1}

\section{Filtering Process}

We consider here a specific class of system operators $\mathcal{H}$ to model the filtering stage, namely those that are linear and space invariant. ${ }^{4)}$ Such systems are commonly assumed in image processing, since the computations can be fully described using linear systems theory. A system is linear if the identity

$$
\mathcal{H}\{a u(x)+b w(x)\}=a \mathcal{H} u(x)+b \mathcal{H} w(x),
$$

holds. Further, a system is space or shift invariant if

$$
\mathcal{H}\left\{u\left(x-x_{0}\right)\right\}=v\left(x-x_{0}\right), \quad \text { for } \mathcal{H}\{u(x)\}=v(x),
$$

to denote that the system operator response is position invariant given identical input conditions. Taken together, the system response for an arbitrary input is fully defined by the correspondence

$$
\mathcal{H}\{u(x)\}=H(x) * u(x), \quad \vdash \quad \mathcal{H}\{\hat{u}(\boldsymbol{k})\}=\hat{H}(\boldsymbol{k}) \cdot \hat{u}(\boldsymbol{k}),
$$

where the left-hand side denotes the convolution of the input signal $u(x)$ by the system's impulse response function $H(x)$ (* is the convolution operator). The correspondence (denoted by $\vdash$ ) establishes that the same result can be computed in the spatial as well as the spectral, or Fourier, domain. In the frequency domain $k$ the convolution is equivalent to a multiplication of the Fourier transforms of the respective signals. This property is useful to study the characteristics of systems.

\subsection{2}

\section{Linear and Space-Invariant Filters}

\subsubsection{1}

\section{Gaussian}

Smoothing for noise suppression is a key operation in early signal processing. An ideal low-pass filter $T \cdot \operatorname{rect}(k T)$ is defined by a specific cut-off frequency $1 /(2 T)$ in the spectral domain. Due to the similarity theorem the corresponding spatial

4) Space invariance is the generalization of the time invariance property defined for time-series analysis. 
filter $\operatorname{si}(\pi x / T)$ (where $\operatorname{si}(x)=\sin (x) / x)$ is of infinite extent with a damping that is proportional to the maximum frequency. In order to utilize a filter function that is localized in the spatial as well as the frequency domain, a Gaussian low-pass is often employed

$$
H^{\text {Gauss }}(x)=\frac{\exp \left(-\|x\|_{2}^{2} / 2 \sigma^{2}\right)}{(\sqrt{2 \pi} \sigma)^{d}} \quad \vdash \quad \hat{H}^{\text {Gauss }}(k)=\exp \left(-\frac{\|k\|_{2}^{2}}{2 \hat{\sigma}^{2}}\right) .
$$

The Fourier transform pair results in two Gaussians which are related by their standard deviations, namely $\hat{\sigma}=1 / \sigma$. Therefore a sharp spatial Gaussian corresponds to a flat Gaussian in the Fourier space and vice versa. (compare Figure 10.9a and e). Due to the Gaussian damping of higher spatial frequencies the filter application reduces the effective resolution of an image, resulting in a coarser scale (see Section 10.4.2).

\subsubsection{2}

\section{First-Order Derivative}

Spatial derivative operations can also be formulated by filtering operations. For example, the first-order derivative is denoted by

$$
u\left(x+e_{j} \mathrm{~d} x\right)=u(x) \mathrm{d} x^{0}+\frac{\partial}{\partial x_{j}} u(x) \mathrm{d} x^{1}+O\left(\mathrm{~d} x^{2}\right), \quad j=1 \ldots d
$$

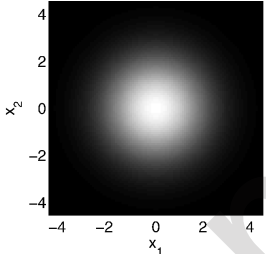

(a)

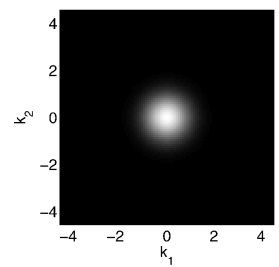

(e)

Fig. 10.9 Filters and their spectra: (a) Gaussian filter. (b) Negative of Laplacian of Gaussian. (c) Odd part of Gabor filter (sine). (d) Even part of Gabor filter (cosine). In all Gaussians $\sigma=1.5$, and for the Gabor filters $\lambda=2, \psi=15 \mathrm{deg}$. The corresponding spectra of the filters are drawn in the lower part.

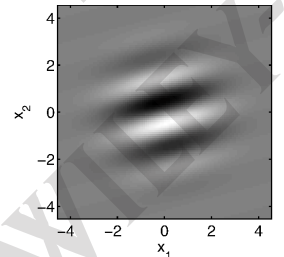

(c)

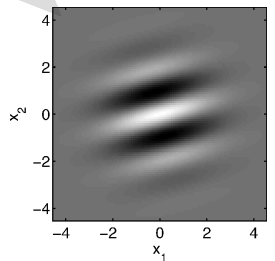

(d)

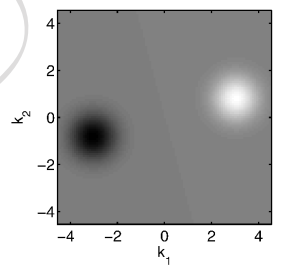

(g)

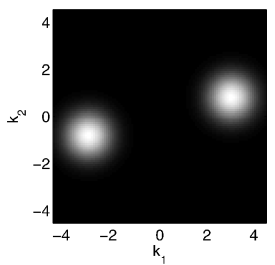

(h)

(e) shows the lowpass filter characteristic of the Fourier transformed Gaussian. ( $f$ ) is a characteristic bandpass filter. (g) visualizes the spatial selectivity of the odd part in the Fourier space, and $(\mathrm{H})$ for the even part. Note that, for better visibility, all values are rescaled to the full intensity range. 
where $e_{j}$ denotes the $j$-th unit vector. Division by $\mathrm{d} x$ and rearranging terms results in the operator for the first-order derivative

$$
\mathcal{H}\{u(x)\}=\frac{u\left(x+e_{j} \cdot \mathrm{d} x\right)-u(x)}{\mathrm{d} x}+O(\mathrm{~d} x) .
$$

All first-order partial derivatives for $j=1, \ldots, d$ together form the gradient of the input image. In Fourier space the derivatives

$$
\mathcal{H}\{u(x)\}=\frac{\partial}{\partial x_{j}} u(x) \quad \vdash \quad \mathcal{H}\{\hat{u}(k)\}=i k \hat{u}(k) \quad j=1 \ldots d,
$$

lead to a multiplication of the original spectrum with $i k$, as the partial derivatives can be calculated within the second integral of (10.15). Multiplication of the spectrum with a linear or faster-than-linear function amplifies noise. This effect can be reduced by appliance of a Gaussian filter kernel before calculating the derivative of the input image.

\subsubsection{3}

\section{Second-Order Derivative and Laplacian of Gaussian (LoG)}

The second-order derivatives are defined on the basis of the Hessian matrix

$$
\mathcal{H}\{u(x)\}=\frac{\partial^{2}}{\partial x_{j} \partial x_{l}} u(x)+\mathcal{H}\{\hat{u}\}=-k_{j} k_{l} \hat{u}(k) \quad j, l=1 \ldots d .
$$

In Fourier space the Hessian matrix is the negative of the transformed image $\hat{u}(k)$ multiplied by the two frequency components $k_{j}$ and $k_{l}$. In this case noise is amplified twice which makes the second-order derivatives highly sensitive, especially for high-frequency noise.

The trace of this Hessian matrix defines the Laplacian operator $\mathcal{L}=\operatorname{trace}\left(\left\{\partial^{2} /\right.\right.$ $\left.\left.\left(\partial x_{j} \partial x_{l}\right)\right\}_{j, l=1 \ldots n}\right)$. For suppression of noise again the Gaussian filter could be applied, before the Laplacian. Due to the law of associativity the convolution of an image with the Laplacian operator can be applied directly to the Gaussian, resulting in the Laplacian-of-Gaussian (LoG)

$$
\begin{aligned}
H^{\mathrm{LoG}}(x) & =\mathcal{L}\left\{H^{\text {Gauss }}(x)\right\}=\frac{1}{(\sqrt{2 \pi} \sigma)^{d}}\left(\frac{\|x\|_{2}^{2}}{\sigma^{4}}-\frac{1}{\sigma^{2}}\right) \exp \left(-\frac{\|x\|_{2}^{2}}{2 \sigma^{2}}\right) \\
& \vdash \hat{H}^{\mathrm{LoG}}(\boldsymbol{k})=-\|\boldsymbol{k}\|_{2}^{2} \hat{H}^{\mathrm{Gauss}}(\boldsymbol{k}),
\end{aligned}
$$

which is characteristic for a bandpass filter, where the frequency with maximal amplification is $\tilde{k}_{j}= \pm \sqrt{2} \hat{\sigma}$ for each dimension $j$. The $2 \mathrm{D}$ version of this filter defines a ring with radius $\sqrt{2} \hat{\sigma}$ of maximum spectral sensitivity (see Figure 10.9f).

\subsubsection{4}

\section{Gabor Filter}

While the LoG operator specifies an isotropic band-pass filter, often orientation sensitive filter devices are needed, for example, to separate oriented texture patterns of 
similar wavelength properties. Gabor filters specify an example in which a selected set of frequencies are passed which fall into the region of a pair of Gaussian windows positioned along a given axis of orientation shifted in opposite directions with respect to the origin (see Figure $10.9 \mathrm{~g}$ and $\mathrm{h}$ ). The combined frequency/phase shift (defined by the wavelength $\lambda$ and direction $\psi$ ) of the Gaussian filter in the frequency domain leads to a modulation of the space domain Gaussian by the wave function $\exp \left(i k_{0} x\right)$.

$$
H^{\text {Gabor }}(x)=\exp \left(i k_{0} x\right) H^{\text {Gauss }}(x), \quad \vdash \quad \hat{H}^{\text {Gabor }}(\boldsymbol{k})=\hat{H}^{\text {Gauss }}\left(\boldsymbol{k}-\boldsymbol{k}_{0}\right) .
$$

Note, that the Gabor filter results in a quadrature filter with an odd (sine) shown in Figure 10.9 (c) and an even part (cosine) shown in (d), ${ }^{5}$ with their corresponding Fourier transforms in (g) and (h), respectively. For the interpretation of these two parts, again the phase and amplitude as defined in (10.14) are considered. Here, the amplitude can be interpreted as the power of the filtered image. The phase has high responses for parts of the image which are coincident with this phase and the specified wavelength. A separated analysis of the two parts shows that the odd part (sine) behaves in a similar way to a bandpass filter, where the even part (cosine) has a Direct Current (DC) level component, due to the residual response

$$
\mathrm{DC}\left(H^{\text {Gabor,even }}\right)=\frac{\int_{\mathbb{R}^{d}} 1 \cos \left(k_{0} x\right) H^{\text {Gauss }}(x) \mathrm{d} x}{(\sqrt{2 \pi} \sigma)^{d}}=\exp \left(-\frac{\left\|k_{0}\right\|_{2}^{2}}{2 \hat{\sigma}^{2}}\right),
$$

for a constant signal. For a DC-level free definition the constant value $\mathrm{DC}\left(H^{\text {Gaboreven }}\right)$ is subtracted from the even part of the Gabor filter, which can be recognized in Figure $10.9 \mathrm{~d}$ by a slightly darker gray in the display of responses as in c, especially for high frequencies.

\subsubsection{5}

\section{Gabor Filter Bank}

Using properly scaled and shifted Gaussian window functions the whole frequency domain could be sampled using Gabor filters. This, in turn, leads to a signal representation by a population of Gabor filter responses (compare Figure 10.10). This sampling can be achieved in two ways. (i) The Gaussian envelope $\hat{\sigma}_{l}$ is constant in all rings; and (ii) the number of Gaussians in each ring is constant, meaning that $\Delta \psi_{l}$ is constant in all rings $l$. For this second approach the wavelengths and standard deviations are

$$
\lambda_{l+1}=\lambda_{l} \frac{1-2 \sin (\Delta \psi / 4)}{1+2 \sin (\Delta \psi / 4)}, \quad \text { and } \quad \hat{\sigma}_{l}=\left(4 \pi / \lambda_{l}\right) \sin (\Delta \psi / 4), \quad l \geq 0,
$$

where $l$ denotes the number of the ring, given $\lambda_{0}$ the radius of the innermost ring. This scheme constructs Gabor wavelets, defined by a constant number of waves in

5) The Hilbert transform $\hat{u}(x)=u(x) * 1 /(\pi x)$ of the even part results in the negative odd part and the Hilbert transform of the odd part results in the even part. 


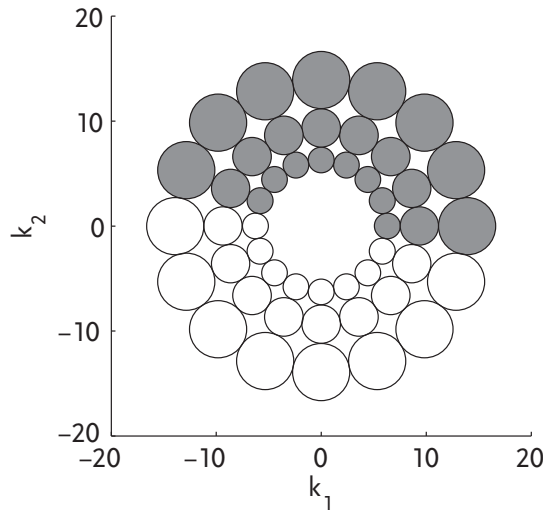

(a)

Fig. 10.10 Construction of Gabor space: (a) Gabor filters drawn as circles with radius of standard deviation for three rings with $\Delta \psi=\pi / 8$, and $\lambda_{0}=1$. Applications using only the energy of the quadrature Gabor filter need only a sampling of the half-space (drawn in gray). Here the whole space (including all

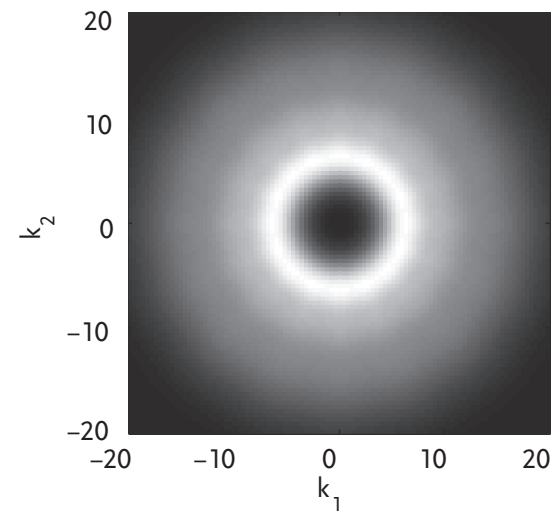

(b)

circles) is sampled. (b) The superimposition of all filter spectra for the even part (cosine). The three filter rings in the total spectrum are visible through the three stepped gray valued regions. For better visibility the square-root of the spectra is shown.

the envelope of the Gaussian for each ring (self-similarity), due to $\lambda_{l+1} / \lambda_{l}=\sigma_{l} / \sigma_{l+1}$. In Figure 10.10 three rings of Gabor filters sampling the Fourier space are depicted. An application of Gabor filters is given in Section 10.5.4 for the extraction of contour lines from images.

\subsection{3}

\section{Morphological Filtering}

In addition to the linear position-invariant filtering, we briefly present a class of nonlinear operations based on mathematical morphology. Such nonlinear filters follow the general schema

$$
\mathcal{H}\{u(x)\}=\mathcal{F}\{\{u(x) \mid x \in \mathcal{N}(x)\}\},
$$

where $\mathcal{F}$ operates on a set and returns a single element of this set, and $\mathcal{N}(x)$ is the neighborhood or support for the operator. These filters are also known as rank order or morphological operators, operating on the order of the elements in the set. For the first filter this set of input values is sorted and the central element is selected by the operator $\mathcal{F}$, which is the median of the input data set. This filter is appropriate for eliminating impulsive noise, visualized in Figure 10.11. In general, this filter obtains edges and transforms patches with continuous gray-level ramps into areas of a single gray-level, caused by the selection of the median element. Morphological operators select the minimum or maximum from the set. Therefore, closed objects become smaller according to their spatial extent for selecting the minimum and, respectively, wider for the maximum selection. These 


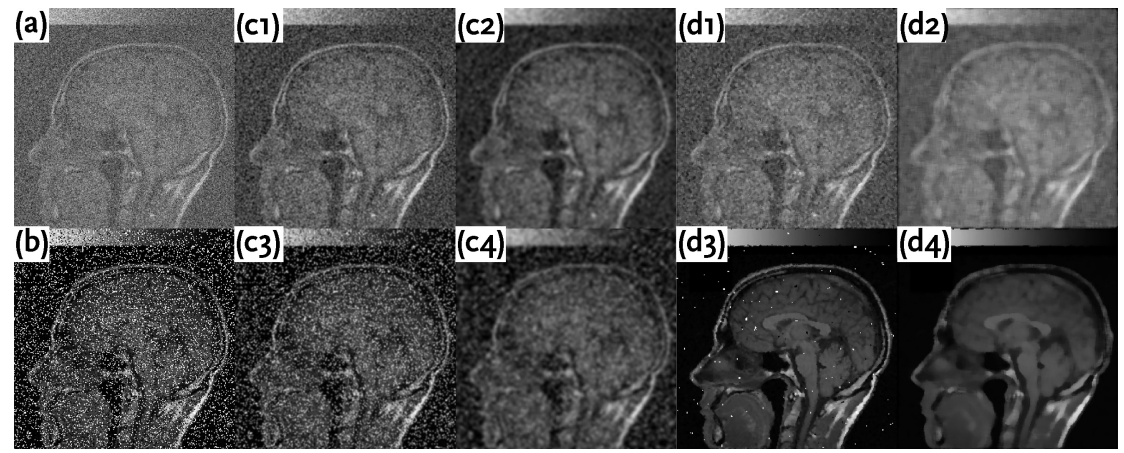

Fig. 10.11 Gaussian and median filter: A magnetic resonance image is distorted by Gaussian noise with standard deviation $\sigma=0.25$ and mean $\mu=0.5$ (a), and impulsive noise where $25 \%$ of all pixels are disturbed (b). Results of filtering with a Gaussian kernel with $\sigma=0.25$ and length of $3 \mathrm{px}$ are shown in (c1) for Gaussian noise and (c3) for outlier noise, and with a Gaussian kernel with $\sigma=1.75$ and length of $7 \mathrm{px}$ in (c2) and (c4), respectively. Results for median filter with a neighborhood of $3 \times 3$ px for Gaussian noise are in (d1) and outlier noise in (d3), and with a neighborhood of $7 \times 7$ px for Gaussian noise in (d2) and outlier noise in (d4). Note that the median filter is appropriate to cope with impulsive noise, and the Gaussian filter is appropriate for handling Gaussian noise.

operations can be consecutively combined resulting in an opening or closing of structures. Further details for opening and closing are reported in Section 10.6.2.

\section{5 .4}

\section{Extraction of Image Structures}

The gradient and higher-order derivatives of an image are key approaches for the extraction of image structures. For the gradient, first-order derivatives (as stated in (10.30)) are calculated. Each position in the image contains a gradient directed into the direction of the strongest increasing gray-value ramp (see Figure 10.12b). An analysis of the structure based on this gradient is not appropriate because uncorrelated noise and a constant gray value patch cannot be distinguished. Thus, the orientation which best fitts the gradients in a neighborhood (for example, defined by the size of the patches) should be calculated. This could be defined as an optimization problem,

$$
\int_{\mathcal{N}\left(x_{0}\right)}\left\|v\left(x_{0}\right) \nabla u(x)\right\|_{2}^{2} \mathrm{~d} x=v\left(x_{0}\right)^{t}\left[\int_{\mathcal{N}\left(x_{0}\right)}(\nabla u)^{t} \nabla u \mathrm{~d} x\right] v\left(x_{0}\right) \stackrel{v}{\rightarrow} \max .
$$

The vector product of the gradient integrated in the local neighborhood is the structure tensor

$$
\{J(u)\}_{j, l}:=\int_{\mathcal{N}\left(x_{0}\right)} u_{x_{j}} u_{x_{l}} \mathrm{~d} x, \quad j, l=1 \ldots d,
$$

for all positions $x_{0}$. The eigenvalue decomposition of $J$ is denoted by the eigenvalues $\lambda_{k}$ and eigenvectors $\boldsymbol{v}_{k} \in \mathbb{R}^{d}$ for $k=1 \ldots d$. The main direction in the neighborhood is the eigenvector corresponding to the largest eigenvalue. For the 2D case the full interpretation of the structure tensor is given in Table 10.2. 


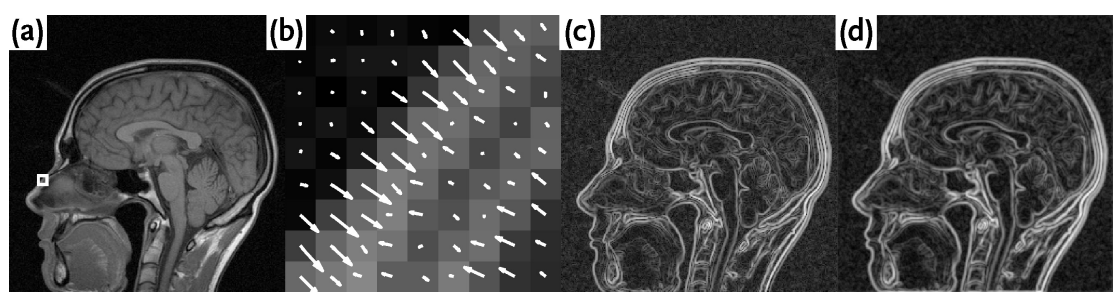

Fig. 10.12 Image gradient, edges and contour: (a) Magnetic resonance image with marked region. (b) Intensity gradient in marked region. (c) Magnitude of intensity gradient. (d) Contour constructed of responses for oriented Gabor filters ( $\sigma=1$, $\Delta \psi=\pi / 8, \lambda=\pi / 3$ ). For clarity in (c) and (d) the square- root is shown.

Tab. 10.2 Interpretation of the structure tensor for a 2D image.

Condition Interpretation

$\lambda_{1} \approx \lambda_{2} \approx 0$

$\lambda_{1} \gg 0, \lambda_{2} \approx 0$

constant gray-value in neighborhood

$\lambda_{1}>\lambda_{2}, \lambda_{2} \gg 0$ directed structure in neighborhood (hint for edge) changes of gray-values in more directions (hint for corner)

Based on the structure tensor, measures for edges and corners in images can be defined. A corner is present if both eigenvalues are significantly greater than zero. A measure considering this condition is the Förstner corner detector [17]. An edge can be identified by the ratio between the eigenvalues. A contour line similar to edges is constructed with a small Gabor filter bank, consisting of one scale and eight orientations, only using the amplitude of the complex filter responses. From this ensemble of filter responses corresponding to each specific orientation the sum is calculated, resulting in the contour signal. This sum is depicted in Figure $10.12 \mathrm{~d}$.

\section{6}

\section{Invariant Features of Images}

Above, we have discussed some basic processing approaches for noise suppression, signal restoration, the detection of localized structures, and their proper coding. The main aim of signal processing is the extraction of relevant features from images to generate compact descriptions. Such descriptions need to possess certain invariance properties, mainly against transformations such as position, rotation, or scaling. Such descriptions serve as a basis for classification or matching different representations utilizing proper similarity measures. In this section we focus on features and descriptions derived thereof which are relevant for Physical Cosmology. We first address the aim to find matchings between objects using representations which are invariant to translation, rotation and scaling. Afterwards, we leave the direct description of scalar fields and switch to methods of stereography, using 
descriptions of binary fields. We present explicit relations of scalar fields to binary fields and discuss the connectivity of structures by topological classification. Then Minkowski functionals are shown as a full set of shape descriptors which obey additivity and are invariant to translations and rotations. We also present their generalization, namely Minkowski valuations. Finally, we end by illustrating applications in cosmology.

\subsection{1}

\section{Statistical Moments and Fourier Descriptors}

Representations with invariant properties are helpful for finding and matching objects and structures. Important invariance properties are translation, rotation, and scaling. These properties are depicted in Figure 10.8a-d. In the next paragraphs several representations which are invariant for at least some transformations are presented.

10.6.1.1

\section{Statistical Joint Central Moments}

The statistical joint central moments of the two random variables (RV)s $X_{1}$ and $X_{2}$ with the joint probability distribution function $u\left(x_{1}, x_{2}\right)$

$$
\begin{aligned}
\mu_{p, q} & =\left\langle\left(\boldsymbol{X}_{1}-\left\langle\boldsymbol{X}_{1}\right\rangle\right)^{p}\left(\boldsymbol{X}_{2}-\left\langle\boldsymbol{X}_{2}\right\rangle\right)^{q}\right\rangle \\
& =\iint\left(x_{1}-\bar{x}_{1}\right)^{p}\left(x_{2}-\bar{x}_{2}\right)^{q} u\left(x_{1}, x_{2}\right) \mathrm{d} x_{1} \mathrm{~d} x_{2},
\end{aligned}
$$

are invariant with translation. If invariance with scale is additionally required we assume that $\tilde{u}\left(x_{1}, x_{2}\right)=u\left(x_{1} / \alpha, x_{2} / \alpha\right)$. Through simple substitutions in the integrals we see that $\tilde{\mu}_{p, q}=\alpha^{p+q+2} \mu_{p, q}$. If we divide $\tilde{\mu}_{p, q}$ through the zeroth moment to the power of $(p+q+2) / 2$ we obtain

$$
\breve{\mu}_{p, q}=\alpha^{p+q+2} \mu_{p, q} /\left(\alpha^{2} \mu_{0,0}\right)^{p+q+2 / 2}=\mu_{p, q} / \mu_{0,0}^{p+q+2 / 2},
$$

which is invariant with scaling. On the basis of the moments a tensor for the moment of inertia

$$
J=\left(\begin{array}{cc}
\mu_{2,0} & -\mu_{1,1} \\
-\mu_{1,1} & \mu_{0,2}
\end{array}\right)
$$

can be constructed. The orientation of the eigenvector corresponding to the smallest eigenvalue of $J$ is $\Phi=1 / 2 \arctan \left(2 \mu_{1,1} /\left(\mu_{2,0}-\mu_{0,2}\right)\right)$, which is the smallest moment of inertia. This criterion is invariant with scaling and translation, because of the invariance of $\mu_{p, q}$ with translation. The calculation of the ratio causes the invariance with scaling.

10.6.1.2

\section{Fourier Descriptors}

Fourier descriptors are a general method used for the compact description and representation of contours. Therefore, we assume that there exists a parameterized 
description $z(t)=z(t+l T) \in \mathbb{C}, t \in[0, \ldots, T], l \in \mathbb{Z}$ of the contour, which is periodic in $T$, and $t$ is the parameter for the actual position of the curve. For the Fourier transformation assume $z(t)=x_{1}(t)+i x_{2}(t)$, that the first component of the curve defines the real part and the second component defines the complex part. The Fourier transform

$$
Z(v)=\frac{1}{T} \int_{0}^{T} z(t) \exp \left(\frac{-2 \pi i v t}{T}\right) \mathrm{d} t, \quad v \in \mathbb{Z}
$$

provides the Fourier coefficients $Z(v)$ for the curve. The first coefficient $Z(0)$ is the mean point or centroid of the curve. The second coefficient $Z(1)$ describes a circle. Including the coefficient $Z(-1)$, a arbitrarily ellipse can be constructed. For each pair of Fourier coefficients $Z(n)$ and $Z(-n)$ an ellipse is constructed, which is run through $n$ times. The reconstruction of the original parameter curve with the Fourier coefficients is

$$
z(t)=\sum_{v=-\infty}^{+\infty} Z(v) \exp \left(\frac{-2 \pi i v t}{T}\right) .
$$

Note that, in practical applications, for appropriate results only a small number of Fourier coefficients must be calculated. Now we consider the invariance properties of this representation. A translational shift in the contour only influences the Fourier coefficient $Z(0)$. A scaling of the contour line influences all coefficients. The same holds for a rotation of the curve. An invariant representation can be constructed in three steps. (i) Drop the coefficient $Z(0)$, which gives translational invariance. (ii) Set the norm of $Z(1)$ to unity, which gives invariance for arbitrary scaling. (iii) Set all phases in relationship to the phase of $Z(1)$, which gives rotational invariance.

In summary, Fourier coefficients are a good representation of contours and moments for the total intensities of objects.

In the following sections we leave the direct description of a scalar field and discuss methods of stereography, particularly binary fields which only have the field value 0 or 1 . This leads to methods of shape description, which we shall discuss later.

10.6.2

\section{Stereography and Topology}

First, we present several definitions and basic methods of stereography. Then we discuss the topological classification of structures which measures their connectivity. Furthermore this gives a motivation for the next subsection.

\subsubsection{1}

\section{Stereography}

To analyze a scalar field $u(x)$ using $x \in \mathbb{R}^{d}$ with methods of stereography one has to generate a binary image called a structure $Q$. This can be done by thresholding. 
One gets the excursion set

$$
Q_{v}:=\{x \mid u(x) \geq v\}
$$

by discriminating between regions with a higher and lower field value than the threshold $v$. The boundary $\partial Q_{v}$ of the excursion set $Q_{v}$ is then obviously given by $\partial Q_{v}:=\{x \mid u(x)=v\}$. Varying the threshold $v$ causes in general a variation in the monochrome image $Q_{v}$. So the threshold can be used as a diagnostic parameter to analyze the scalar field $u(x)$.

Given a structure $Q$ or even only a point distribution, that is a union of points which can also be understood as a structure $Q$ one can generate the parallel set $Q_{\varepsilon}$. By putting a ball $B_{\varepsilon}$ with fixed radius $\varepsilon$ at every point of the structure $Q$ one gets

$$
Q_{\varepsilon}=Q \oplus B_{\varepsilon}
$$

The sum is understood as the Minkowski sum $C=A \oplus B$ of two sets $A$ and $B$, which consists of all points $\boldsymbol{c}=\boldsymbol{a}+\boldsymbol{b}$ that can be written as a vector sum of two points $\boldsymbol{a} \in A$ and $\boldsymbol{b} \in B$. The corresponding difference $Q \ominus B_{\varepsilon}$ is called the Minkowski difference. In image processing these operations are called dilation and erosion. Again varying the radius $\varepsilon$ causes, in general, a variation of the generated structure $Q_{\varepsilon}$. Therefore the radius $\varepsilon$ can also be used as a diagnostic parameter.

Note that, in general, $\left(Q \oplus B_{\varepsilon}\right) \ominus B_{\varepsilon} \neq Q \neq\left(Q \ominus B_{\varepsilon}\right) \oplus B_{\varepsilon}$ holds true. Both have an effect of smoothing on a length scale $\varepsilon$. Closing is understood as $Q_{\varepsilon}^{\oplus, \ominus}=\left(Q \oplus B_{\varepsilon}\right) \ominus B_{\varepsilon}$ and opening as $Q_{\varepsilon}^{\ominus, \oplus}=\left(Q \ominus B_{\varepsilon}\right) \oplus B_{\varepsilon}$ where, compared to $Q$, the structure $Q_{\varepsilon}^{\oplus, \ominus}$ loses small holes and $Q_{\varepsilon}^{\ominus, \oplus}$ loses small cusps. As discussed in Section 10.5.3, effects of closing and opening can also be achieved by applying nonlinear and space-invariant filters on scalar fields.

Another way to get a diagnostic parameter to analyze a scalar field $u(x)$ is to apply an appropriate filter before thresholding. Then the individual filtering parameters can be used as diagnostic parameters. In practice, it is useful to restrict oneself to one diagnostic parameter which reflects the feature of interest and hold the other parameters fixed. For filter processes we refer to Sections 10.5.1-10.5.3.

\subsubsection{2}

\section{Topology}

A useful feature to distinguish between different structures is their connectivity. To analyze the connectivity of a structure $Q$ we use the topological measure called the Euler Characteristic (EC), denoted by $\chi$, which is related to the genus $g$ by $\chi=1-g$. The definition we present here is not only motivated by historical reasons from set theory and convex geometry, but also provides a good access to its interpretation. For a convex body $K$ the EC is defined by

$$
\chi(K):= \begin{cases}1 & \text { for } K \neq \emptyset \\ 0 & \text { for } K=\emptyset\end{cases}
$$

and obeys the functional equation for adding two convex bodys $K_{1}$ and $K_{2}$

$$
\chi\left(K_{1} \cup K_{2}\right)=\chi\left(K_{1}\right)+\chi\left(K_{2}\right)-\chi\left(K_{1} \cap K_{2}\right) \quad \text { and } \quad \chi(c K)=\chi(K),
$$


the scaling property, for scaling a convex body $K$ with a constant positive real number $c \in \mathbb{R}^{+}$.

There is a demonstrative morphological interpretation of the value of the EC which is governed by the number $N(\diamond)$ of objects with the characteristic $\diamond$ in the structure $Q$. For 2D structures, there are $\chi(Q)=N$ (components) $-N($ holes $)$. Positive values are generated by isolated objects of a spot-like structure and negative values point to a mesh-like structure, where the absolute value reflects the strength. For 3D structures, there are $\chi(Q)=N$ (components) $+N$ (cavities) $-N$ (tunnels). If there is a connected structure then positive values reflect a cheese-like structure and negative values a sponge-like structure. The absolute value again reflects the strength. Figure 10.13 illustrates the functional equation of the EC in (10.48) and its interpretation for 2D structures.

Given a smooth $d \mathrm{D}$ structure $Q$ with $d>1$ and a regular boundary $\partial Q$, then every point $x \in \mathbb{R}^{d}$ on its hypersurface has $d-1$ principal curvature radii $R_{i}(x)$ with $i=1, \ldots, d-1$. The local mean curvature $H$ and Gaussian curvature $G$ of the hypersurface are defined by

$$
H:=\frac{1}{d-1} \sum_{i=1}^{d-1} \frac{1}{R_{i}(\mathbf{x})} \quad \text { and } \quad G:=\prod_{i=1}^{d-1} \frac{1}{R_{i}(\mathbf{x})} .
$$

Its EC follows from the Gauss-Bonnet theorem after surface integration

$$
\chi(Q)=\frac{\Gamma(d / 2)}{2 \pi^{d / 2}} \int_{\partial Q} G \mathrm{~d} A
$$

where $\mathrm{d} A$ denotes the element of the hypersurface $\partial Q$ and $\Gamma(x)$ the $\Gamma$-function. The EC of a $d \mathrm{D}$ excursion set $Q$ follows from the Morse theorem by counting the different stationary points of the thresholded function which lie in the excursion set. It is

$$
\chi\left(Q_{v}\right)=\sum_{k=0}^{d}(-1)^{k} N_{k}\left(Q_{v}\right),
$$

where $N_{k}\left(Q_{y}\right)$ denotes the number of stationary points $(\nabla u=0)$ to the index $k$, where $k$ is the number of negative eigenvalues of the matrix $\left\{\partial_{i} \partial_{j} u\right\}$ for every stationary point. For a 2D excursion set $Q_{y}$, which was generated from a function $u(x)$ with $x \in \mathbb{R}^{2}$, we get $\chi\left(Q_{y}\right)=N($ maxima $)+N($ minima $)-N$ (saddle points), where now $N(\diamond)$ denotes the number of stationary points of kind $\diamond$ which are in the excursion set [19].

In practice, the EC is a appropriate measure to study percolation, wetting and connectivity where only the topology is of interest. To study also the geometry of structures one needs more measures. This leads to Minkowski functionals and their generalization, Minkowski valuations, which will be studied in the next section. 


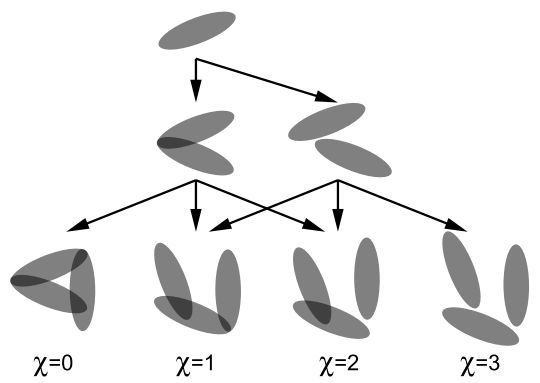

Fig. 10.13 Euler Characteristic (EC). Multiple union (adding from top to bottom following the arrows) of convex bodies (light gray) illustrates the continuation of the functional equation of the EC in (10.48). The intersections are shown by dark gray coloration. Comparing the columns shows the interpretation of the EC for $2 \mathrm{D}$ structures, i.e. the value of the EC reflects the number of components minus the number of holes [18].

10.6.3

\section{Minkowski Functionals and Minkowski Valuations}

Minkowski functionals (MFs) permit are to analyze the morphology, that is, the topology and geometry of structures. They map these structures on numbers or, in a generalized way, on tensors which will be discussed later in the case of Minkowski valuation (MVs), with a well-known geometric and topological meaning. Historically, MFs were introduced in convex geometry by Hermann Minkowski and were called "Quermaßintegrale". Appropriations in stochastic geometry and integral geometry followed [20-22].

\subsubsection{1}

\section{Stochastic Geometry}

If one wants to analyze the morphology of a $d \mathrm{D}$ structure, one needs appropriate measures which map the structure $Q$ on a set of values $F(Q)$. For simplicity let these values be real numbers, then the mapping can be formulated by

$$
Q \rightarrow F_{j}(Q) \in \mathbb{R} \text { for } j=0, \ldots, n \text {. }
$$

Because we are only interested in the morphology of the structure $Q$ we can make a few assumptions for the mapping and, respectively, the functional $F(Q)$. Thus the number $n$ of linear independent functionals $F_{j}(Q)$ may be quantified as follows.

(I) Additivity: the functional behaves as a volume, in a mathematical sense like the functional equation of the EC in (10.48),

(ii) Motion invariance: the mapping is independent of the position and orientation of the structure, that means that the functionals are independent on applying translations and rotations to the structure $Q$.

(iii) Conditional continuity: if a structure $Q_{1}$ is continuous in a structure $Q_{2}$ then also the functional $F\left(Q_{1}\right)$ is continuous in the functional $F\left(Q_{2}\right)$.

One gets, from the Functional theorem of stochastic geometry which was found by Hugo Hadwiger [20], that a $d \mathrm{D}$ structure has $d+1$ linearly independent functionals which satisfy (i)-(iii). Others follow by linear combination. One full set of these descriptors are the MFs which represent intuitive parameters in common dimensions (see Table 10.3). Note that there is the freedom of scaling them by positive real numbers $c \in \mathbb{R}^{+}$. In reasonable applications, there is a need for normalization. 
Tab. 10.3 Geometrical and topological interpretation of the $d+1$ Minkowski Functionals of structures in common dimensions $d=1,2,3$.

$d=1 \quad d=2 \quad d=3$

\begin{tabular}{llll}
\hline$F_{0}$ & length & area & volume \\
$F_{1}$ & Euler Characteristic & circumference & surface area \\
$F_{2}$ & - & Euler Characteristic & total mean curvature \\
$F_{3}$ & - & - & Euler Characteristic
\end{tabular}

In any normalization the homogeneity of MF

$$
F_{j}(c Q)=c^{d-j} F_{j}(c Q) \quad \text { for } \quad j=0, \ldots, d
$$

of a $d \mathrm{D}$ structure $Q$ holds true. This is consistent with the scaling property of the $\mathrm{EC}$ in (10.48).

\subsubsection{2}

\section{Integral Geometry}

With this interpretation in mind we can focus on an integral geometric approach which is suitable for the description of a smooth $d \mathrm{D}$ structure $Q$ with $d>1$ and a regular boundary $\partial Q$. This approach leads to a natural generalization of the framework by calculating higher moments. For this reason we add an extra upper index in the notation of the MFs in (10.54) to show that they are tensors of rank 0 , namely scalars.

The MF of a structure $Q$ for $j=0$ follows by a volume integration and a set of $d$ MFs for $j=1, \ldots, d$ by a surface integration

$$
F_{j}^{0}(Q)=\left\{\begin{array}{cc}
\mathcal{N}_{0}^{0} \int_{Q} \mathrm{~d} V & \text { for } \quad j=0 \\
\mathcal{N}_{j}^{0} \int_{\partial Q} S_{j} \mathrm{~d} A & \text { for } j=1, \ldots, d .
\end{array}\right.
$$

$\mathrm{d} V$ denotes the hypervolume element of the $d \mathrm{D}$ structure $Q$ and $\mathrm{d} A$ the hypersurface element of its $(d-1) \mathrm{D}$ surface. The integrands of a set of the $d$ MFs for $j=1, \ldots, d$ can be generated by the $j$-th elementary symmetric function $S_{j}$, which is defined by

$$
\sum_{j=1}^{d} z^{d-j} S_{j}:=\prod_{i=1}^{d-1}\left[z+\frac{1}{R_{i}(\mathbf{x})}\right] .
$$

The functions $S_{j}$ follow by comparing the coefficients of the polynomial in $z$ and are functions of the $j-1$ principal curvature radii $R_{j-1}(x)$ at the position $x \in \mathbb{R}^{d}$. With the definitions in (10.49) one can see that for $d=2$ we have $S_{1}=1$ and $S_{2}=G$. For $d=3$ we have $S_{1}=1, S_{2}=H$ and $S_{3}=G$ and for $d>3$ always $S_{1}=1, S_{2}=H$ and $S_{d}=G$. Note the consistency between the statements in Table 10.3. The prefactors 
$\mathcal{N}_{j}^{0}$ for $j=0, \ldots, d$ are arbitrary but fixed normalization constants as explained before are caused by the freedom of normalization [22].

Compared to scalar MFs, one finds that tensor-valued MFs, further called Minkowski Valuations (MVs) to distinguish clearly between MFs, also obey additivity (i) and conditional continuity (iii). But motion invariance (ii) breaks down. Therefore, MVs obey motion covariance and the number $n$ of linear independent functionals $F_{j}(Q)$ with $j=0, \ldots, n$ can again be quantified. Note that, for rank $r>1$ in $d$ dimensions, $n$ differs from $d+1$ and $n \leq d+r-1$ holds true [23].

By adding the position vector $\mathbf{x} \in \mathbb{R}^{d}$ in the integrands in (10.54) one gets

$$
F_{j}^{1}(Q)=\left\{\begin{array}{ccc}
\mathcal{N}_{0}^{1} \int_{Q} \mathbf{x} \mathrm{d} V & \text { for } & j=0 \\
\mathcal{N}_{j}^{1} \int_{\partial Q}^{Q} S_{j} \mathbf{x} \mathrm{d} A & \text { for } & j=1, \ldots, d
\end{array}\right.
$$

for the first-order MVs. Due to the multiplication by a $d \mathrm{D}$ vector, the mapping in (10.52) now reads $Q \rightarrow F_{j}^{1}(Q) \in \mathbb{R}^{d}$ for $j=0, \ldots, d$. Therefore these MVs become $d \mathrm{D}$ vectors, that are first-rank tensors.

As mentioned before, there is the possibility of constructing more than $d+1$ linear independent MVs of rank $r>1$. Second-order MVs can be constructed using

$$
F_{j}^{2}(Q)=\left\{\begin{array}{ccc}
\mathcal{N}_{0}^{2} \int_{Q} \mathbf{x}^{2} \mathrm{~d} V & \text { for } & j=0 \\
\mathcal{N}_{j}^{2} \int_{\partial Q} S_{j} \mathbf{x}^{p} \mathbf{n}^{q} \mathrm{~d} A & \text { for } j=1, \ldots, d+r-1
\end{array}\right.
$$

where $\boldsymbol{n} \in \mathbb{R}^{d}$ denotes the normalized normal vector on the hypersurface $\partial Q$ in the point $x$ and $p, q \in \mathbb{N}_{0}$ with rank $r=p+q=2$. The multiplication of the $d \mathrm{D}$ vectors in the integrands is understood as a symmetric tensor product, which for two $d \mathrm{D}$ vectors $\boldsymbol{a}=\left(a_{1}, \ldots, a_{d}\right)$ and $\boldsymbol{b}=\left(b_{1}, \ldots, b_{d}\right)$ leads to a $d \times d$ matrix with the elements $c_{i j}=a_{i} b_{j}(i, j=1, \ldots, d)$. The mapping in (10.52) now reads $Q \rightarrow F_{j}^{2}(Q) \in \mathbb{R}^{d \times d}$ for $j=0, \ldots, d+r-1$. Therefore these MVs get second-rank tensors with $d \times d$ elements.

Although higher-rank tensors can be constructed, we will not consider them here. Next, we show several applications of MFs and MVs motivated by cosmological interest.

\subsubsection{3}

\section{Applications}

In practice, MFs and MVs turned out to be robust measures for a huge bandwidth of applications. Due to the intuitive interpretation, the individual measures can be related to some physical properties like the EC for percolation studies. Also full sets of theoretical expected values of MFs for several randomly generated structures are known $[19,21]$. In cosmology, galaxy distributions were studied and compared to several Poisson point processes. Around every point a ball with radius $\varepsilon$ was placed (see (10.46)), where the radius was varied and used as a diagnostic parameter. This technique is known as the boolean Germ-Grain-Model.

In cosmology random fields also play an important role. Density fluctuations of the very early universe as imprinted in the CMB, are assumed to be Gaussian. 
To check the Gauss hypothesis, which is a fundamental aspect to allow highlyprecision cosmology and is the basis for simulations, MFs are used [18, 22, 24]. MFs of a thresholded Gaussian random field $Q v$ (see (10.45)) are analytically well known $[19,22]$ being

$$
F_{j}^{0}\left(Q_{v}\right)=\left\{\begin{array}{clc}
\mathcal{N}_{0}^{0}[1-\Phi(v-\mu / \sqrt{2 \sigma})] & \text { for } & j=0 \\
\mathcal{N}_{j}^{0} H_{j-1}(v-\mu / \sqrt{\sigma}) & \text { for } \quad j=1, \ldots, d,
\end{array}\right.
$$

where

$$
\Phi(x)=2 / \sqrt{\pi} \int_{0}^{x} \mathrm{~d} t \exp \left(-t^{2}\right) \quad \text { and } \quad H_{n}(x)=(-1)^{n} / \sqrt{2 \pi}(\mathrm{d} / \mathrm{d} x)^{n} \exp \left(-x^{2} / 2\right)
$$

is the Gaussian error function $\Phi(x)$ and $H_{n}(x)$ the $n$th Hermite function. These only depend on parameters of the stochastic process, that is, the mean $\mu$ and the variance $\sigma$ of the field $u(x)$ and not of the cosmological process, in particular, the cosmological parameters. Depending on the application it can be more convenient to simulate a huge set of realizations including additional numerical effects like discretization, pixelation and masking, also dealt with by experimental data. Further, one then also gets the statistical variance to perform likelihood analyses and one can define a confidence level. Applications to the CMB are shown in Figure 10.14.

Similarly, the evolution of the LSS of the universe, from its Gaussian origin to its current observed net-like structure, can be studied by MFs of the total field. Since high thresholds disentangle the cosmic web by yielding many isolated objects the concept of shapefinders, which provide measures to distinguish between different shape characteristics of single objects, is useful. An illustrative example in 2D, actually on the sphere, as used for an analysis of hot and cold spots in the CMB [18], is to quantify the elongation of structures being approximately ellipses by the ratio of their area to their squared circumference $\mathcal{E}(Q):=F_{0}^{0}(Q) /\left[F_{1}^{0}(Q)\right]^{2}$. This is a dimensionless, and thereby scale-invariant, shapefinder $\mathcal{E}$, which immediately provides an axis ratio for expected shapes.

Let us come back to the example of the LSS in 3D. Three independent ratios of MFs which have the dimension of length, namely thickness $\mathcal{T}$, width $\mathcal{W}$ and length $\mathcal{L}$, can be defined:

$$
\mathcal{T}(Q):=\frac{F_{0}^{0}(Q)}{2 F_{1}^{0}(Q)}, \quad \mathcal{W}(Q):=\frac{2 F_{1}^{0}(Q)}{\pi F_{2}^{0}(Q)} \quad \text { and } \quad \mathcal{L}(Q):=\frac{3 F_{2}^{0}(Q)}{4 F_{3}^{0}(Q)}
$$

An appropriate normalization is $\mathcal{N}_{0}^{0} \equiv 1, \mathcal{N}_{1}^{0} \equiv 1 / 6, \mathcal{N}_{2}^{0} \equiv 1 / 6 \pi$ and $\mathcal{N}_{3}^{0} \equiv 1 / 2 \pi$. Then for every convex body $K$ the property $\mathcal{L}(K) \geq \mathcal{W}(K) \geq \mathcal{T}(K)$ holds true. Further dimensionless shapefinders called the

$$
\text { planarity } \quad \mathcal{P}(Q):=\frac{\mathcal{W}-\mathcal{T}}{\mathcal{W}+\mathcal{T}} \text { and filamentarity } \mathcal{F}(Q):=\frac{\mathcal{L}-\mathcal{W}}{\mathcal{L}+\mathcal{W}}
$$




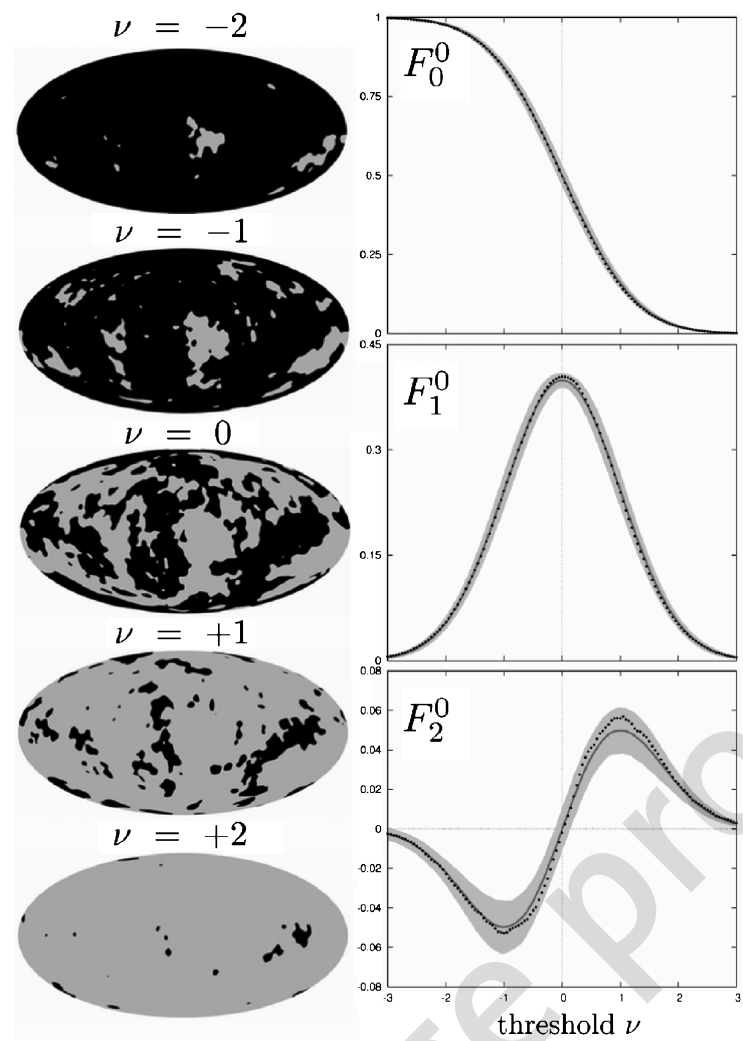

(a)

(b)

Fig. 10.14 Minkowski Functionals (MFs). Test on Gaussianity of the fluctuations of the cosmic microwave background (CMB) with Minkowski Functionals (MFs). The normalized signal became thresholded (as illustrated in (a), where the analyzed structure is the black area). The corresponding normalized MFs $F_{0}^{0}$, $F_{1}^{0}$ and $F_{2}^{0}$ are calculated (black dots in graphs in (b)) and compared to the mean values (dark gray line) and statistical variances ( $3 \sigma$ as light gray area) of a Gaussian random field. The area functional $F_{0}^{0}$ is equivalent to the cumulative normalized height distribution and therefore drops monotonously. By increasing the threshold, holes appear, get bigger and become connected. Isolated spots appear, get smaller and finally vanish. Therefore the length functional $F_{1}^{0}$ increases, reaches a maximum and decreases again. Negative values of the connectivity functional $F_{2}^{0}$ reflect the appearance of holes, and positive values the appearance of isolated spots, where in the intermediate both balance each other. With these results likelihood analysis showed a high confidence level for the CMB being a Gaussian random field [18].

can be defined, providing appropriate measures to discriminate between LSS of different evolution scenarios of physical modeling and their comparison to real data. Their behavior is demonstrated in Figure 10.15. These shapefinders also provide the possibility of categorizing observed galaxy shapes. A real galaxy differs from a sphere by being an oblate spheroid, (like a pancake) known as the family of spiral galaxies, or being a prolate spheroid, (like a cigar) known as the family of elliptical galaxies [22]. 


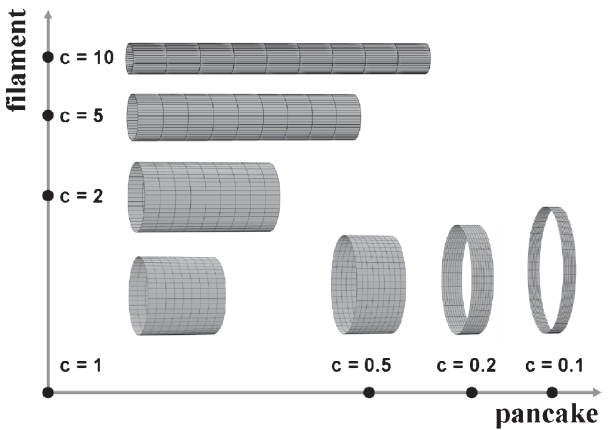

(a)

Fig. 10.15 Shapefinders:. Different cylinders as test bodies (a). For illustration only their cover is shown. The ratio of their height to their diameter is denoted by $c$. Varying $c$ from zero to infinity causes a transition from a pancake to a filament (gray arrows). Note the invariance of scaling. The scatter plot (b) of the corresponding shapefinders planarity $\mathcal{P}$

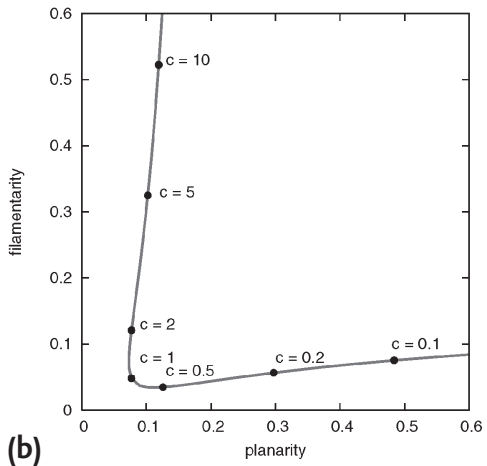

and filamentarity $\mathcal{F}$ (black points). The results of varying $c$ from zero to infinity continuously are shown as a gray line. Building a pancake $(c \rightarrow 0)$ the value of the shapefinder $\mathcal{F}$ stays stable, but $\mathcal{P}$ increases. On the other hand, by building a filament $(c \rightarrow \infty)$ the value of the shapefinder $\mathcal{P}$ stays stable, but now $\mathcal{F}$ increases.

MFs are useful for analyzing structures of a stochastic origin or single objects with $\chi=1$. Given more than one single object, MFs can have identical values for different structures (compare (5) and (6) in Figure 10.16). When the relative position of partial structures is important, as in analysis of the inner structure of galaxies [25], galaxy clusters or galaxy superclusters [26], one can use MVs for a reasonable description. Due to the concept of center of mass and moments of inertia, known from mechanics, a possible interpretation becomes obvious [23]. First-order MVs can be interpreted as the geometrical center of the scalar measure and one can define curvature centroids

$$
\mathbf{p}_{j}(Q)=F_{j}^{1}(Q) / F_{j}^{0}(Q) \text { with } \mathcal{N}_{j}^{0} \equiv \mathcal{N}_{j}^{1} \equiv 1 \text { for } j=0, \ldots, d .
$$

To fulfill the mentioned interpretation of Second-order MVs one considers only a subset of (10.57) with $r=p=2$ and executes an appropriate transformation. Then one gets the elements

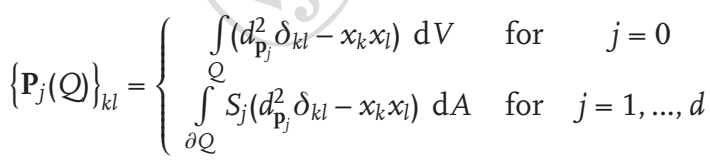

of the curvature tensors $\mathbf{P}_{j}(Q)$ for every corresponding scalar measure. $d_{\mathbf{p}_{j}}^{2}$ is the distance to the corresponding curvature centroid $\mathbf{p}_{j}$, which is used as the origin of the coordinate system, and $\delta_{k l}$ is the Kronecker $\delta$. These tensors satisfy the eigenvalue equation

$$
\mathbf{P}_{j}(Q) \mathbf{v}_{j}^{m}(Q)=\lambda_{j}^{m}(Q) \mathbf{v}_{j}^{m}(Q) \text { for } j=0, \ldots, d \text { and } m=1, \ldots, d
$$

with $\lambda_{j}^{m}(Q) \in \mathbb{R}, \mathbf{v}_{j}^{m}(Q) \in \mathbb{R}^{d}$ and $\mathbf{v}_{j}^{m}(Q) \cdot \mathbf{v}_{j}^{n}(Q)=0$ for $m \neq n$. Thus the scalar measures of the structure $Q$ were vectorized, which means parameterized, by the 
(1)

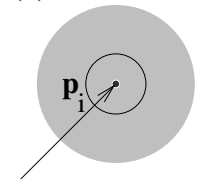

(4)

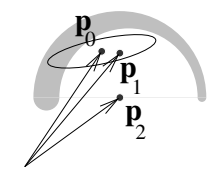

(2)

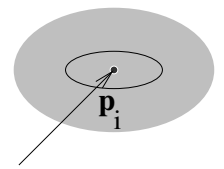

(5)

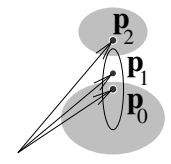

(3)

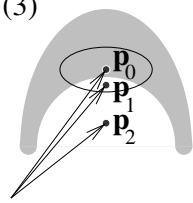

(6)

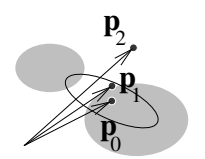

Fig. 10.16 Minkowski Valuations (MVs). Some simple structures (gray), their curvature centroids $\mathbf{p}_{i}$ with $i=0,1,2$ and their area tensor $\mathbf{P}_{0}$ (black ellipse, which indicates the ratio of eigenvalues and eigendirections). A circle (1) and an ellipse (2) with the same volume. Because of point symmetry, all centroids coincide, but due to orientation and elongation, the tensors differ. Given an axis-symmetric structure which is not point-symmetric (3) the centroids no longer coincide, but still remain in one straight line, which indicates, as well as the tensor, the symmetry axis. Breaking additional axis symmetry is shown in (4). The scalar Minkowski functional cannot distinguish between structure (5) and (6). Tensor Minkowski valuations on the other hand can. (Reprinted Figure 5 from [23] with kind permission of Springer Science and Business Media. We thank the author Claus Beisbart for providing the data for reproduction.).

orientation and strength in orthogonal directions along the direction of the eigenvectors $\mathbf{v}_{i}^{m}(Q)$ and their corresponding eigenvalues $\lambda_{j}^{m}(Q)$. Figure 10.16 illustrates the power of the shape description with MVs on a number of simple 2D structures.

These measures immediately serve as descriptors or, adjusted to the application combinations, as in the case of MFs in the concept of shapefinders, are more advantageous. Let us restate the task of quantifying the elongation of structures being approximately ellipses. For an ellipse $E$, where $a$ and $b$ denote the two semi-axes, $a / b=\sqrt{\lambda_{0}^{1}(E) / \lambda_{0}^{2}(E)}$ when $a \geq b$ then $\lambda_{0}^{1}(E) \geq \lambda_{0}^{2}(E)$ holds true. Again an appropriate shapefinder is found. Combining this with the one stated before in the case of MFs, it even provides the possibility of defining quality measures for expected shapes [18].

\section{7}

\section{Concluding Remarks}

This work was initiated by the observation that, in both research areas, namely Computer Vision and Physical Cosmology similar tasks in image analysis are employed. Therefore, we have highlighted several methodological approaches concerning topics of Computer Vision and Physical Cosmology in the field of image processing. The aim was to give some examples that show how different disciplines arrive at related approaches that can be considered at a more systemic level of a classical processing hierarchy. 
Motivated by the recording of the cosmic microwave background (CMB) on the celestial sphere, we started with projection methods, in particular, the Mollweide projection (see Figure 10.2). Next, we discussed the representation of images and characterized four main properties, namely the space an image can be defined on, the quantization, the resolution and the scale space of intensities. Scale spaces are known from physics, but here the properties of scale spaces were adapted to methods of image processing according to [11].

Images and their characteristics were not only defined in the plane, but also on arbitrary surfaces (see Figure 10.3a). For example, the two-point correlation function was defined on the sphere and, in addition, the angular power spectrum. This angular power spectrum is a measure used to compare the expected CMB for different models of the Universe with the measured CMB (see Figure 10.6).

After consideration of image characteristics, the basic methods in image processing, modeled by a filtering process, were discussed. Besides the analysis of simple filters like the Gaussian or the Laplacian of the Gaussian, we additionally discussed the Gabor filter and specified a scheme for the construction of a Gabor filter bank (see Figure 10.10). On the basis of partial derivatives of image intensities an intensity gradient was constructed. This gradient denotes the steepest gray-value ramp. A structure tensor based on the gradient was defined, containing information of the local gray-value distribution. For the 2D case, an interpretation of this structure tensor was given (see Table 10.2).

In the last part we focused on invariant descriptions of image features, where invariance was restricted to scaling, translation and rotation. First, statistical moments for continuously valued images and descriptions of contour lines were described. Therefore, the contour lines were assumed to be defined as periodic curves and a representation based on Fourier coefficients was employed. Then we changed to thresholded images, denoted by binary structures which could be characterized by the Euler Characteristic as a simple measure. But, for the analysis of the topology and geometry of structures, this measure was not sufficient, therefore scalar Minkowski functionals were considered. These measures are suitable to analyze structures initiated by a random process, like investigating the statistical properties of the CMB (see Figure 10.14) or for shape recognition of single objects by means of shapefinders (see Figure 10.15). Also scalar Minkowski functionals do not reveal the full description of structures as explained in Figure 10.16. Thus, we introduced tensor Minkowski valuations offering a more detailed analysis of structures but also leaving motion invariance (translation and rotation).

In summary, we have outlined the primal methods and general concept of image analysis. Generally, these methods are located within the first steps of an imageprocessing hierarchy, providing image enhancement and basic feature extraction normally seen as low- and mid-level vision tasks. Based on these first steps, further methods like 3D scene reconstruction, optic flow estimation, or classification and identification, can be realized. In particular, for the last two tasks the given invariant descriptions are essential. In conclusion, basic methods and general concepts 
will also be useful in other research areas besides image processing, but so far, a starting point has been given for an exchange of problems and existing solutions among researchers studying our observable world.

\section{References}

1 Ballard, D.H. and Brown, C.M. (1982) Computer Vision, Prentice-Hall

2 Forsyth, D.A. And Ponce, J. (2003) Computer Vision - A Modern Approach, 3rd ed, Pearson Education International, Upper Saddle River, New Jersey.

3 Ullman, S. (1996) High-Level Vision, MIT Press, Cambridge, Massachusetts.

4 Witkin, A.P. and Tenenbaum, J.S. (1983) On the role of structure in vision, in Human and Machine Vision (eds J. Beck, B. Hope and A. Rosenfeld), Academic Press.

5 Faugeras, O. (1993) Three-dimensional Computer Vision, MIT Press, Cambridge, Massachusetts.

6 Einstein, A. (1917) Kosmologische Betrachtungen zur allgemeinen Relativitätstheorie. Sitzungsberichte der Preußischen Akademie der Wissenschaften 1917 S. 142-152 (also in: The Collected Papers of Albert Einstein Bd. 6 S. $540 \mathrm{ff}$ Princeton University Press 1996).

7 Spergel D.N. et al. (2007) Three-year Wilkinson Microwave Anisotropy Probe (WMAP) observations: Implications for cosmology. The Astrophysical Journal Supplement Series 170, 377-408.

8 Górski, K.M., Hivon, E., Banday, A.J., Wandelt, B.D., Hansen, F.K., Reinecke, M. AND Bartelmann, M. (2005) HEALPix: A framework for high-resolution discretization and fast analysis of data distributed on the sphere. The Astrophysical Journal 622, 759-771.

9 Gomes, J., Darsa, L., Costa, B. And VelHo, L. (1999) Warping and Morphing of Graphical Objects, Morgan Kaufmann Publishers, San Francisco.

10 Hilbert, D., Cohn-Vossen, S. (1952) Geomtery and Imagination, Chelsea Publ. Company, New York.

11 Weickert, J. (1998) Anisotrope Diffusion in Image Processing, Teubner Verlag, Stuttgart.

12 Horn, B.K.P. (1986) Robot Vision, MIT Press, Cambridge, Massachusetts.

13 Trucco, E. ANd Verri, A. (1998) Introductory Techniques for 3-D Computer Vision, Prentice-Hall, Upper Saddle River, New Jersey.

14 Aurich, R., Janzer, H.S., Lustig, S. And Steiner, F.() Do we live in a ,small Universe“?. arXiv:0708.1420, accepted for publication in Classical and Quantum Gravity, 2007/Ts

15 Fischer, B. ANd Modersitzki, J. (2006) Image Fusion and Registration - a Variational Approach. In Computational Science and High Performance Computing II, Notes on Numerical Fluid Mechanics and Multidisciplinatory Design, Springer, 193205.

16 Jäнne, B. (2005) Digital Image Processing, 6th edn, Springer Verlag Berlin, Heidelberg, New York.

17 FÖRSTNER, W. (1986) A feature based correspondence algorithm for image matching. ISP Comm. III, Rovaniemi 1986, International Archives of Photogrammetry, 26-3/3.

18 JANZER, H.S. (2006) Untersuchung der kosmischen Hintergrundstrahlung mit Minkowskifunktionalen. Diploma thesis, Universität Ulm. 
19 TомiтA, H. (1990) Statistics and Geometry of Random Interface Systems. In Formation, Dynamics and Statistics of Patterns (Kawasaki, K., Suzuki, M., and Onuki, A. eds), volume 1, World Sientific.

20 Hadwiger, H. (1957) Vorlesungen über Inhalt, Oberflüche und Isoperimetrie. Springer.

21 Меске, K.R. (1996) Integralgeometrie in der statistischen Physik: Perkolation, komplexe Flüssigkeiten und die Struktur des Universums. Verlag Harri Deutsch.

22 Schmalzing, J. (1999) On Statistics and Dynamics of Cosmic Structure. Doctoral dissertation, LMU München.

23 Beisbart, C., Dahlke, R., Mecke, K. AND WAGNER, H. (2002) Vector- and Tensor-valued Descriptors for Spatial Patterns, in Morphology of Condensed Matter.
Physics and Geometry of Spatial Complex Systems (eds K. Mecke and D. Stoyan), Springer.

24 Eriksen, H.K., Novikov, D.I., Lilje, P.B., BANDAY, A.J. AND GoRsKI, K.M. (2004) Testing for Non-Gaussianity in the Wilkinson Microwave Anisotropy Probe Data: Minkowski Functionals and the Length of the Skeleton. Astrophys. J. 612, 64-80.

25 Rahman, N. AND Shandarin, S.F. (2003) Measuring shapes of galaxy images - I. Ellipticity and orientation. Monthly Notices of the Royal Astronomical Society 343(3), 933-948.

26 Beisbart, C., Buchert, T. and Wagner, H. (2001) Morphometry of spatial patterns. Physica A 293, 592-604. 